\title{
MAPPING THE SHORES OF THE BROWN DWARF DESERT. II. MULTIPLE STAR FORMATION IN TAURUS-AURIGA
}

\author{
Adam L. Kraus ${ }^{1,5}$, Michael J. Ireland ${ }^{2}$, Frantz Martinache ${ }^{3}$, and Lynne A. Hillenbrand ${ }^{4}$ \\ ${ }^{1}$ Institute for Astronomy, University of Hawaii, 2680 Woodlawn Dr., Honolulu, HI 96822, USA \\ ${ }^{2}$ Sydney Institute for Astronomy (SIfA), School of Physics, University of Sydney, NSW 2006, Australia \\ ${ }^{3}$ National Astronomical Observatory of Japan, Subaru Telescope, Hilo, HI 96720, USA \\ ${ }^{4}$ Department of Astrophysics, California Institute of Technology, MC 249-17, Pasadena, CA 91125, USA \\ Received 2010 August 16; accepted 2011 January 10; published 2011 March 17
}

\begin{abstract}
We have conducted a high-resolution imaging study of the Taurus-Auriga star-forming region in order to characterize the primordial outcome of multiple star formation and the extent of the brown dwarf desert. Our survey identified 16 new binary companions to primary stars with masses of $0.25-2.5 M_{\odot}$, raising the total number of binary pairs (including components of high-order multiples) with separations of 3-5000 AU to 90 . We find that $\sim 2 / 3-3 / 4$ of all Taurus members are multiple systems of two or more stars, while the other $\sim 1 / 4-1 / 3$ appear to have formed as single stars; the distribution of high-order multiplicity suggests that fragmentation into a wide binary has no impact on the subsequent probability that either component will fragment again. The separation distribution for solar-type stars $\left(0.7-2.5 M_{\odot}\right)$ is nearly log-flat over separations of 3-5000 AU, but lower-mass stars $\left(0.25-0.7 M_{\odot}\right)$ show a paucity of binary companions with separations of $\gtrsim 200 \mathrm{AU}$. Across this full mass range, companion masses are well described with a linear-flat function; all system mass ratios $\left(q=M_{B} / M_{A}\right)$ are equally probable, apparently including substellar companions. Our results are broadly consistent with the two expected modes of binary formation (free-fall fragmentation on large scales and disk fragmentation on small scales), but the distributions provide some clues as to the epochs at which the companions are likely to form.
\end{abstract}

Key words: binaries: general - brown dwarfs - stars: low-mass - stars: pre-main sequence

Online-only material: color figures, machine-readable tables

\section{INTRODUCTION}

The frequency and properties of multiple star systems offer powerful constraints on star formation and early cluster evolution. For example, the newest generation of theoretical models now broadly match the slope and turnover of the initial mass function (IMF; e.g., Bate 2009a). Simultaneous agreement with the mass-dependent frequency, separation distribution, and mass-ratio distribution for binary systems is a far more demanding criterion, and one that has yet to be achieved. The ubiquity of binary systems suggests that an understanding of multiple star formation is also necessary to truly understand other processes such as cluster formation, protoplanetary disk evolution, and planet formation.

The past two decades have seen numerous studies of nearby field binary systems in order to constrain their frequency and properties. These surveys (e.g., Duquennoy \& Mayor 1991, hereafter DM91; Fischer \& Marcy 1992, hereafter FM92; Close et al. 2003; Bouy et al. 2003; Burgasser et al. 2003; Raghavan et al. 2010) have found that binary frequencies and properties are very strongly dependent on mass. Solar-mass stars have high binary frequencies $(\gtrsim 60 \%)$, the separation distribution appears to be log-normal with a peak of $\sim 30 \mathrm{AU}$ and includes binary stars with separations of $>10^{4} \mathrm{AU}$, and the mass-ratio distribution includes many low-mass companions. By contrast, very low mass (VLM) stars and brown dwarfs have a low binary frequency $(\sim 20 \%)$, small mean separations $(\sim 4 \mathrm{AU})$ and maximum separations $(\lesssim 20 \mathrm{AU})$, and a strong tendency to have equal-mass companions. The form of the mass-dependent transition between these regimes is still unclear for field stars,

\footnotetext{
5 Hubble Fellow.
}

though there is some evidence for a smooth transition (e.g., FM92; Reid \& Gizis 1997).

Parallel surveys of young star-forming regions have supported some of these conclusions, but also indicated intriguing differences. In surveys of Class II/III T Tauri stars in sparse associations like Taurus and Upper Scorpius, the companion frequency is very high ( $\gtrsim 80 \%$; Ghez et al. 1993; Simon et al. 1995; Köhler et al. 2000; Kraus et al. 2008). This trend does not seem to hold for denser young clusters such as IC 348 and the ONC (Duchêne et al. 1999; Köhler et al. 2006; Reipurth et al. 2007) and old open clusters such as $\alpha$ Per, the Pleiades, and Praesepe (Bouvier et al. 1997; Patience et al. 2002), where the binary frequency is similar to that of field stars; it is still unclear whether this difference is a primordial feature caused by different initial conditions or an evolutionary feature resulting from dynamical interactions. Sparse associations also have a much higher frequency of wide binary companions than either dense clusters or the field (Kraus \& Hillenbrand 2007a, 2009a; Reipurth et al. 2007), a difference that most likely does result from dynamical disruption of wide binary systems in the latter populations. This strongly argues that neither clusters nor the field represent a dynamically pristine population, and therefore they provide limited constraints on the binary formation process. By contrast, sparse associations seem to represent a more primordial population. Observations of younger Class $0 / \mathrm{I} T$ Tauri stars suggest that binary properties evolve as protostars are assembled out of their natal cores (e.g., Duchêne et al. 2004; Haisch et al. 2004; Connelley et al. 2008), so even results for Class II/III systems include the migration and dynamical interactions that occur after fragmentation; as we discuss further in Section 6, some of the properties of these slightly older systems could offer hints regarding very early evolution. 
Our theoretical expectations for this young population are still highly uncertain. Several models have been proposed as the primary mechanism for multiple star formation (e.g., Bonnell 2001; Tohline 2002), with the three leading contenders being prompt fragmentation during an isothermal protostellar clump's initial free-fall collapse, fission of a nonisothermal protostellar core after free-fall collapse has ended, and disk fragmentation after the primary star has condensed and acquired a massive accretion disk. Fission seems to have been ruled out by hydrodynamical simulations, as a collapsing nonisothermal core will evolve on the Kelvin-Helmholtz timescale and angular momentum seems to be transported to the extremes of a nonaxisymmetric core at a much faster pace (e.g., Durisen et al. 1986; Bonnell 1994). However, prompt fragmentation and disk fragmentation remain as viable explanations for different types of binary formation.

The most widely accepted model for the formation of wide ( $>100$ AU) binary systems is by prompt fragmentation, during or just after the epoch where the prestellar core has become Jeans critical and begun free-fall collapse, but before the core has become nonisothermal and heating acts to oppose further collapse (as reviewed by Bodenheimer \& Burkert 2001). This process typically is modeled using smoothed particle hydrodynamic (SPH) simulations (Bate 2000; Bate et al. 2002; Delgado-Donate et al. 2004); the most recent simulations of larger-scale star formation implicitly include this process by extending down to much smaller angular scales ( $\sim 5 \mathrm{AU}$; Bate 2009a; Offner et al. 2009). After a protostellar core undergoes sufficient collapse to form a central protostar, the remaining envelope accretes into a circumstellar disk and from the disk onto the star. If the disk accumulates material from the envelope more quickly than mass can accrete onto the star, then it could grow more massive and violate the Toomre stability criterion (Toomre 1964), fragmenting to form a bound companion. This process has been modeled extensively for the formation of extrasolar planets (e.g., Boss 2001), but if there is sufficient material left in the disk and envelope, this bound companion would then accrete additional mass and grow into a stellar binary companion (e.g., Clarke 2009). Observations suggest that the characteristic radius for a protostellar disk and for accretion onto it is 50-100 AU (Enoch et al. 2009; Watson et al. 2007), so disk fragmentation could explain binary formation at small scales where prompt fragmentation is not feasible. Neither model has yielded quantitative predictions to date, but as we discuss in Section 6, we can use the predicted trends from these models and the observed properties of binary systems to infer some basic conclusions for multiple star formation.

One specific topic of recent interest is the formation of substellar binary companions. Over the past 15 years, radial velocity (RV) surveys have discovered many short-period stellar companions and exoplanets, but relatively few companions with masses of 10-80 $M_{\text {Jup }}$ (e.g., Marcy \& Butler 2000; Grether \& Lineweaver 2006), a gap known as the "brown dwarf desert." Coronagraphic imaging surveys for wide companions have suggested that substellar companions might not be unusually rare, but instead could have a frequency consistent with an extension of the binary mass-ratio function (e.g., Metchev \& Hillenbrand 2009). However, neither survey technique has been able to study the 5-50 AU regime, a separation range which represents the peak of the binary separation distribution for solar-type binaries, as well as the giant planet regime for our own solar system. Substellar companions bridge the mass range between binaries and exoplanets, so a census in this unexplored regime could indicate whether the substellar companion mass function and separation distribution more closely resemble the stellar or planetary cases. This census would also reveal the origin of the RV brown dwarf desert; while it is possible that substellar companions never form at all, the paucity at small separations could also be traced to secondary effects like inefficient migration.

In this paper, we present a high-resolution imaging survey of the Taurus-Auriga star-forming region. Our survey uses adaptive optics (AO) and aperture-masking interferometry to achieve unprecedented angular resolution and depth, yielding a more complete view of the primordial multiple star population and the so-called brown dwarf desert. In Section 2, we describe our survey sample, and in Section 3, we summarize the observations and our data analysis techniques. We summarize our new observational results for Taurus-Auriga and place them in the context of past surveys in Section 4, and then in Section 5, we characterize the binary properties for solar-type stars. Finally, in Section 6, we use these results to infer the processes and timescale and lengthscale of multiple star formation.

\section{SURVEY SAMPLE}

The member census of Taurus-Auriga has been assembled gradually over the past several decades. The extremely low stellar density and variable extinction make it difficult and expensive to survey the association, especially away from the central cores. The wide range of evolutionary stages (from Class 0 protostars to Class III diskless stars) also results in a wide range in member properties, requiring numerous observing techniques to achieve completeness. We compiled a then-current (though still incomplete) stellar census in two previous works (Kraus \& Hillenbrand 2007a, 2008), and have used that census as the basis for our aperture-masking sample. Many additional members have been identified by Luhman et al. (2006, 2009), Scelsi et al. (2008), and Rebull et al. (2010), but most are VLM stars or brown dwarfs that fall below the mass range of our survey.

Our original census selected every known member that had been shown to have at least one signature of youth (i.e., infrared excess, accretion, typical lithium abundance for 1-3 Myr old stars, or low surface gravity). We also explicitly required every member to have a known spectral type, so that we could infer a mass and study the mass dependence of measured properties. This requirement rejected most of the very young Class $0 / \mathrm{I}$ sources, leaving only the more evolved Class II/III sources. The selection of our observed sample was subject to several biases. Natural guide star AO observations can be conducted only with a guide star that is optically bright $(R \lesssim 15$, with a brighter limit under marginal observing conditions). This requirement yields an effective joint limit in mass and extinction. The AO correction is also compromised for binary pairs with similar brightness $(\Delta R \lesssim 1-2)$ and moderately close separations $\left(\sim 1^{\prime \prime}-4^{\prime \prime}\right)$ since both sources are imaged on the wavefront sensor. Finally, we are unable to use aperture masking for the components of known binaries with separations of $\sim 0.0^{\prime \prime} 4-1^{\prime \prime} .0$ because they are close enough for their interferograms to overlap, but too widely separated for the power spectrum to yield unaliased measurements.

In Table 1, we list all of the Taurus members that passed a preliminary spectral type cut $\left(\mathrm{G} 0 \leqslant \mathrm{SpT} \leqslant \mathrm{M} 4\right.$, or $2.5 M_{\odot} \gtrsim M \gtrsim$ $0.25 M_{\odot}$ according to the methods described in Section 3.3) and have optical/NIR fluxes which are not dominated by scattered light (i.e., obscured by a circumstellar envelope, as for Class 0/I 
Table 1

Stars in Taurus-Auriga with SpT $\leqslant$ M4

\begin{tabular}{|c|c|c|c|c|c|c|c|}
\hline \multirow[t]{2}{*}{ Name } & R.A. & Decl. & \multirow[t]{2}{*}{$\mathrm{SpT}$} & \multirow{2}{*}{$\begin{array}{c}R \\
(\mathrm{mag})\end{array}$} & \multirow{2}{*}{$\begin{array}{c}K \\
(\mathrm{mag})\end{array}$} & \multirow[t]{2}{*}{ Group $^{a}$} & \multirow[t]{2}{*}{ References } \\
\hline & \multicolumn{2}{|c|}{ (J2000) } & & & & & \\
\hline \multicolumn{8}{|c|}{ Observed members } \\
\hline 2M04080782 & 40807.82 & 280728.0 & M3.75 & 15.0 & 11.39 & $\mathrm{P}$ & 1 \\
\hline LkCa 1 & 41314.14 & 281910.8 & M4 & 12.6 & 8.63 & $9, \mathrm{H}$ & 2 \\
\hline Anon 1 & 41327.23 & 281624.8 & M0 & 12.1 & 7.46 & $\mathrm{~F}$ & 2 \\
\hline FM Tau & 41413.58 & 281249.2 & M0 & 13.3 & 8.76 & $12, \mathrm{~L}$ & 2 \\
\hline CW Tau & 41417.00 & 281057.8 & $\mathrm{~K} 3$ & 12.3 & 7.13 & 8 & 2 \\
\hline FP Tau & 41447.31 & 264626.4 & M4 & 12.7 & 8.87 & 11 & 2 \\
\hline CX Tau & 41447.86 & 264811.0 & M2.5 & 12.6 & 8.81 & $10, \mathrm{~K}$ & 2 \\
\hline $\mathrm{LkCa} 4$ & 41628.11 & 280735.8 & K7 & 11.6 & 8.32 & 6 & 2 \\
\hline CY Tau & 41733.73 & 282046.9 & M1 & 12.4 & 8.60 & 8 & 2 \\
\hline LkCa 5 & 41738.94 & 283300.5 & M2 & 12.5 & 9.05 & 9 & 2 \\
\hline
\end{tabular}

Notes. Positions and $K$ magnitudes are from 2MASS, $R$ magnitudes are from NOMAD (Zacharias et al. 2004). Magnitudes are uncertain by $\sigma_{R} \sim 0.25 \mathrm{mag}$ and $\sigma_{K} \sim 0.03 \mathrm{mag}$. Spectral types and membership are determined from the references listed.

${ }^{a}$ Our survey targets were observed in groups, such that single targets could be used as calibrators for newly discovered binaries. We also list the composition of these groups in Table 2.

b Short-period spectroscopic binary, as characterized by Mathieu et al. (1997) for DQ Tau AB, Prato et al. (2002) for UZ Tau Aab, White \& Hillenbrand (2005) for StHa 34 Aab, and Massarotti et al. (2005) for V826 Tau AB. For our analysis, the component masses of spectroscopic binaries will be combined and treated as a single higher mass.

References. (1) Luhman 2006; (2) Kenyon \& Hartmann 1995; (3) Strom \& Strom 1994; (4) G. Herczeg 2010, private communication; (5) White \& Hillenbrand 2004; (6) Duchêne et al. 1999; (7) Hartigan et al. 1994; (8) Kraus \& Hillenbrand 2008; (9) White \& Hillenbrand 2005; (10) Briceño et al. 1999; (11) Briceño et al. 1998; (12) Hartigan \& Kenyon 2003; (13) White et al. 1999; (14) White \& Basri 2003; (15) Schaefer et al. 2009; (16) Luhman \& Rieke 1998; (17) Luhman 2000; (18) Kenyon et al. 1998; (19) Briceño et al. 2002; (20) Luhman et al. 2003a; (21) Martín 2000; (22) Slesnick et al. 2006; (23) Luhman 2004; (24) NOMAD (Zacharias et al. 2004); (25) White \& Ghez 2001; (26) Stapelfeldt et al. 2003.

(This table is available in its entirety in a machine-readable form in the online journal. A portion is shown here for guidance regarding its form and content.)

sources, or an edge-on disk). The spectral type range was chosen to match the nominal upper end of the Taurus mass function (which has $<5$ known A-F stars) and to avoid strong incompleteness for stars which are too faint for AO observations (with

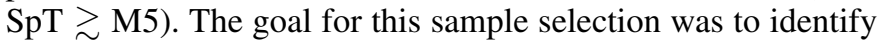
a sample of low-mass stars that are analogs of field solar-type or early-M stars.

The stars in Table 1 are divided between the observed sample (82 targets), known binaries that we did not re-observe (37 targets), and the stars that we could not observe (many of which have other, less sensitive multiplicity observations available). This last group is comprised of all stars fainter than $R=15$ (16 targets) and the similar-flux, moderately wider binary pairs that have $\Delta R<2$ and separations $<4^{\prime \prime}$ (five targets). For binary systems with fainter secondaries, we tried when possible to simultaneously observe both components; otherwise, we concentrated on the primary. We also observed six stars that passed our observational selection criteria but have spectral types of $<\mathrm{G} 0$ or $>\mathrm{M} 4$, two stars that appear to be nonmembers (HBC 352 and HBC 353; Kraus \& Hillenbrand 2009b), two $1^{\prime \prime}-2^{\prime \prime}$ binary companions that were serendipitously observed in the same images as their primary (StHa 34 B and RW Aur B), and one Class I source that served as a test for our ability to distinguish companions from extended emission (HL Tau). These stars are not included in our statistical analysis since the vast majority of Taurus members in those categories could not be observed, but we list these stars in Table 1 and will report their results for completeness.

We ultimately omitted 42 known binary systems, which introduces a bias against the detection of additional binary components that would denote hierarchical triple systems; if the presence of a wide tertiary influences subsequent fragmentation, then our results might not reflect the total population of Taurus.
There are also 12 systems with separations of $1^{\prime \prime}-4^{\prime \prime}$ for which we could observe only the primary. As we discuss further in Section 5.2, much of this incompleteness can be remedied by using Bayesian analysis to infer the parameters of the binary population, but the validity of this correction depends on the degree of independence between wide binary formation and the subsequent fragmentation of their components into close pairs.

We also could not observe three known or suspected edge-on disk systems: Haro 6-5B, HH 30, and V710 Tau C. Furthermore, edge-on disk hosts are more difficult to identify in membership surveys since they do not fall on the association's photometric sequence, so there could be additional Taurus members that remain undiscovered. These two biases lead to some incompleteness for our multiplicity census among all disk hosts, but since the disk inclination is a purely geometric effect, it should not influence our conclusions.

We can partially remedy the incompleteness for unobservable binary systems by adopting the results of previous survey programs. There is a long history of multiplicity programs studying Taurus-Auriga, starting with the lunar occultation and speckle surveys of the 1980s and 1990s, and leading up to modern day searches using speckle imaging as well as natural and laser guide star AO. Many of the previous surveys labored under different selection biases from our own (i.e., NIR flux limits or the presence of tip-tilt guide stars, instead of our optical flux limits), so they complement our own sample and allow for a more complete mass-limited sample. We did not re-observe the known binary systems in order to increase the survey efficiency, and we will adopt the previous detection limits for members we could not observe. In Section 4, we present a census of the known binary systems and of the best detection limits for all apparently single stars. 
Table 2

Observed Groups

\begin{tabular}{|c|c|c|c|c|}
\hline Group & Telescope & Filter & $\begin{array}{l}\text { Epoch } \\
\text { (MJD) }\end{array}$ & Stars \\
\hline \multicolumn{5}{|c|}{ Fall 2007} \\
\hline 1 & Keck & Lp & 54428.3 & RY Tau, SU Aur, HP Tau-G2, AB Aur, HD 283572 \\
\hline 2 & Keck & $\mathrm{Kp}$ & 54427.3 & GM Aur, UX Tau A, LkCa 14, LkCa 19 \\
\hline 3 & $\mathrm{Pal}$ & $\mathrm{Ks}$ & 54432.3 & HBC 388, DR Tau, HBC 427 \\
\hline 4 & Pal & Ks & 54434.3 & V827 Tau, L1551-51, V826 Tau, DN Tau, DS Tau \\
\hline 5 & Pal & Ks & 54434.3 & HBC 352, HBC 353, BP Tau, DG Tau \\
\hline 6 & Keck & $\mathrm{Kp}$ & 54427.3 & LkCa4, DE Tau, LkCa 15, V830 Tau \\
\hline 7 & Keck & $\mathrm{Kp}$ & 54427.5 & GK Tau, GI Tau, DL Tau, Hubble 4 \\
\hline 8 & Keck & $\mathrm{Kp}$ & 54428.4 & CY Tau, CW Tau, IP Tau, HP Tau \\
\hline 9 & Keck & Kp & 54427.4 & LkCa 1, DH Tau, LkCa 5, UZ Tau A \\
\hline 10 & Keck & $\mathrm{Kp}$ & 54427.6 & DM Tau, CX Tau, FF Tau, HP Tau-G3, CoKu Tau/4 \\
\hline 11 & Keck & $\mathrm{Kp}$ & 54428.5 & LkCa 21, HN Tau, FP Tau, DP Tau, DM Tau \\
\hline 12 & Keck & $\mathrm{Kp}$ & 54428.6 & FM Tau, HL Tau, GO Tau, HO Tau \\
\hline \multicolumn{5}{|c|}{ Fall 2008} \\
\hline A & Keck & Lp & 54821.2 & AB Aur, HD 283572, SU Aur, RY Tau \\
\hline B & Keck & $\mathrm{Hc}$ & 54823.4 & AB Aur, HD 283572, SU Aur, RY Tau \\
\hline $\mathrm{C}$ & Keck & $\mathrm{Kp}$ & 54822.2 & UX Tau, GM Aur, LkCa 15, LkCa 14, BP Tau \\
\hline $\mathrm{D}$ & Keck & $\mathrm{Kp}$ & 54823.5 & HP Tau G2, HBC 427, DS Tau, L1551-51, V826 Tau, RX J0507.2+2437 \\
\hline E & Keck & $\mathrm{Kp}$ & 54823.3 & HBC 376, JH 56, DI Tau, AA Tau, L1551-55, DH Tau, V836 Tau \\
\hline $\mathrm{F}$ & Keck & $\mathrm{Kp}$ & 54821.3 & DO Tau, DK Tau, Anon 1, CI Tau \\
\hline $\mathrm{G}$ & Keck & $\mathrm{Kp}$ & 54821.5 & RW Aur A, RW Aur B, RX J05072+2437, IP Tau, HV Tau, V836 Tau \\
\hline $\mathrm{H}$ & Keck & $\mathrm{Kp}$ & 54823.5 & DN Tau, LkCa 1, StHa 34 A, StHa 34 B \\
\hline $\mathrm{J}$ & Keck & Kp & 54821.5 & IQ Tau, V819 Tau, HN Tau, DQ Tau \\
\hline K & Keck & $\mathrm{Kp}$ & 54821.4 & CX Tau, IT Tau, FT Tau, DM Tau \\
\hline $\mathrm{L}$ & Keck & $\mathrm{Kp}$ & 54823.2 & FM Tau, HO Tau, J1-665, J1-507, SCH04390160, HL Tau \\
\hline M & Keck & $\mathrm{Kp}$ & 54822.3 & V410 X-ray 1, FY Tau, FZ Tau, HK Tau, JH 108 \\
\hline $\mathrm{N}$ & Keck & $\mathrm{Kp}$ & 54822.3 & GO Tau, CIDA-9, CIDA-10, CIDA-12, 2M04414565 A \\
\hline $\mathrm{P}$ & Keck & $\mathrm{Kp}$ & 54822.5 & $\begin{array}{l}\text { FN Tau, JH 223, CIDA-8, CIDA-11, 2M04554757, CoKu Tau/3 A, } \\
\text { Haro 6-13, I04187+1927, 2M04080782 }\end{array}$ \\
\hline
\end{tabular}

\section{OBSERVATIONS AND ANALYSIS}

\subsection{Observations}

The technique of non-redundant aperture masking has been well established as a means of achieving the full diffraction limit of a single telescope (e.g., Nakajima et al. 1989; Tuthill et al. 2000, 2006). The core innovation of aperture masking is to resample the telescope's single aperture into a sparse interferometric array; this allows for data analysis using interferometric techniques (such as closure-phase analysis) that calibrate out the phase errors that limit traditional astronomical imaging by inducing speckle noise. As we described in Kraus et al. (2008, hereafter K08), aperture-masking observations can yield contrasts of $\Delta K \sim 6$ at $\lambda / D$ and $\Delta K \sim 4$ at $1 / 3 \lambda / D$, and we used the technique to identify over a dozen binary companions that fall inside the detection limits of traditional imaging surveys. More detailed discussions of the benefits and limitations of aperture masking, as well as typical observing strategies, can be found in the first paper of this series (K08) and in Readhead et al. (1988), Nakajima et al. (1989), Tuthill et al. (2000, 2006), Lloyd et al. (2006), Martinache et al. (2007), and Ireland et al. (2008).

We observed our survey targets over the course of two observing runs at Keck (in 2007 November and 2008 December) and one observing run at Palomar (in 2007 November). All of our targets were observed with the facility AO imagers, Keck/ NIRC2 (K. Matthews et al. 2011, in preparation) and Palomar/ PHARO (Hayward et al. 2001), which have aperture masks installed in cold filter wheels at or near the pupil stop. Most observations were conducted using the $K^{\prime}$ or $K_{s}$ filters, but we observed the brightest targets in both $H$ and $L^{\prime}$ in order to maximize the resolution (in $H$, for close stellar companions) and depth (in $L^{\prime}$, since low-mass companions should be very red). In all cases, we used a nine-hole aperture mask that passes $11 \%$ of the total incident flux through nine $1.1 \mathrm{~m}$ subapertures. The choice maximizes the throughput, as the other option (an 18 hole mask) passes half as much incident flux and can be used only with narrowband filters (due to wavelength-dependent dispersion in broadband filters) that are $\sim 10 \%$ as wide as the corresponding broadband filters.

A typical interferometric measurement requires the observation of one or more source-calibrator pairs. However, our sample included numerous targets with similar positions and brightnesses, so instead, we observed groups of science targets and inter-calibrated between them; we described our observing methodology in more detail in Kraus et al. (2008). We started our survey by including association nonmembers (selected from Two Micron All Sky Survey (2MASS) to have colors consistent with distant giants) as external calibrators. However, we abandoned this tactic partway through the survey because the nonmembers were resolved into binary systems as often as the Taurus members were, which defeated their purpose. We summarize the observations for each group of targets in Table 2.

The observing sequences for these groups were identical to those described in Kraus et al. (2008), with each target being observed in three visits that each consisted of eight exposures with individual exposure time of $20 \mathrm{~s}$. The total integration time per target was $480 \mathrm{~s}$, and including acquisition and observation overheads, the total time required per target was $\sim 15$ minutes. 
All observations in $H$ and $K^{\prime}$ were taken without dithering, such that the interferograms for all targets were placed in the same location on the detector (which was chosen to be free of bad pixels). Our analysis of dithered observations shows that on some telescopes, including different dither positions degrades the calibration (for reasons that we are still investigating), so we decided that it was more important to achieve good calibration for our (relatively bright) targets than to attempt sky subtraction of a background that was typically negligible. The $L^{\prime}$ sky background is much more significant $\left(2500\right.$ counts $\left.\mathrm{s}^{-1}\right)$, so we chose a minimal two-point dither pattern. In this case, there was no evidence that the dithers affected the data analysis.

The observing conditions varied; our 2007 November observing runs were plagued by poor seeing and clouds, but our 2008 December observations were conducted under good to excellent seeing $\left(0.2-0^{\prime} .5\right.$ in the $K$ band). We therefore decided to re-observe those targets from 2007 that had maximum sensitivities of $\Delta K \lesssim 4$ at the separation bin where we typically achieve optimal sensitivity (80-160 mas); the requirement of $\Delta K \gtrsim 4$ insures that typical $1 M_{\odot}$ sample members will have detection limits near the bottom of the brown dwarf mass range $\left(M_{\lim } \lesssim\right.$ $20 M_{\text {Jup }}$ ). We will report both sets of detection limits.

\subsection{Data Analysis and Detection Limits}

The data analysis follows almost the same prescription as in Kraus et al. (2008), so we discuss here only a general background to the technique and differences from Kraus et al. (2008). The data analysis takes three broad steps: basic image analysis (flat-fielding, bad pixel removal, dark subtraction), extraction and calibration of squared visibility and closure phase, and binary model fitting. Unless fitting to close, near-equal binaries, we fit only to closure phase, as this is the quantity most robust to changes in the $\mathrm{AO}$ point-spread function (PSF). We converted the on-chip position angles (P.A.s) to on-sky P.A.s using the most recent NIRC2 P.A. calibration by Ghez et al. (2008), and treated the conversion between different $K$ filters $\left(K^{\prime}\right.$ versus $\left.K_{s}\right)$ as negligible (e.g., Carpenter 2001) compared to the intrinsic uncertainties in relative photometry for $\mathrm{AO}$ observations ( $\sim 0.03-0.05 \mathrm{mag})$.

The final detection limits are found using a Monte Carlo method that simulates 10,000 random closure-phase data sets of a point source with closure-phase errors and covariances that match those of the calibrated target data set. This routine then searches for the best fit for a companion in each randomized data set. Over each annulus of projected separation from the primary star, the $99.9 \%(3.3 \sigma)$ confidence limit (listed in Table 4) is set to the contrast ratio where $99.9 \%$ of the Monte Carlo trials have no best binary fit with a companion brighter than this limit anywhere within the annulus. The validity of this technique was demonstrated empirically by there being no spurious detections in Kraus et al. (2008) above this limit, despite there being several near the limit.

A major difference between the Upper Scorpius analysis of Kraus et al. (2008) and the Taurus analysis in this paper relates to the on-sky P.A.s of the observations. The aperture mask was always used in a vertical angle mode at Keck, meaning the camera coordinates were fixed with respect to the elevation axis of the telescope, rather than being fixed with respect to $\mathrm{N}$ and $\mathrm{E}$. For declinations that differed significantly from the telescope latitude, this meant that the P.A. of the baselines changed with time, synthesizing a larger field of view. However, for Taurus, the declination is similar to the latitude of Keck, so if a target was observed while only rising or only setting, there was no sky rotation for aperture synthesis. We did not observe any targets within $<30$ minutes of transit so that any apparent rotation of a companion during a single integration would be $\ll \lambda / D$.

Previous papers on aperture masking (e.g., Martinache et al. 2007, 2009; Kraus et al. 2008) have shown extracted visibilities and closure phases. We do not repeat this here, but note that it is difficult to plot the raw closure phases because the data are represented as a discrete set of points on a four-dimensional grid. Instead, in Figure 1, we show two representative power spectra (i.e., two-dimensional maps of the visibilities) taken using the nine-hole aperture mask at Keck. The non-redundant geometry of the aperture mask leads to a power spectrum made of individual peaks (also referred to as splodges) corresponding to the baselines sampled by the mask. CIDA-9A shows an elongated core of the interferogram, and power that decreases to the top left and bottom right. However, closure phases are zero within errors, meaning that this star has no close companions with good limits. We can confidently assign the elongation to a symmetrical instrumental cause (windshake in this case). CIDA-10 clearly shows two cores in the interferogram, and a corresponding sinusoidal modulation of the power spectrum amplitude when compared with CIDA-9. An even wider binary system would still have the core of the companion within the interferogram, but would show modulation within a single peak in the power spectrum. To reveal such wide companions, individual splodges need to be subsampled. However, this additional analysis only led to the detection of one additional companion (2M04414565 Aa+Ab).

\subsection{Stellar and Companion Properties}

Stellar properties can be difficult to estimate, particularly for young stars, since pre-main-sequence stellar evolutionary models are not well calibrated. The mass of a given sample could be systematically uncertain by as much as $20 \%$ (e.g., Hillenbrand \& White 2004), and individual masses could be uncertain by factors of two or more due to unresolved multiplicity or the intrinsic variability that young stars often display (from accretion or rotational modulation of star spots). This suggests that any prescription for determining stellar properties should be treated with caution.

We estimated the properties of our sample members using the method described in Kraus \& Hillenbrand (2007a), specifically adapted for stars of the median age of Taurus ( $~ 2 \mathrm{Myr}$; Kraus $\&$ Hillenbrand 2009b). This procedure combines the $2 \mathrm{Myr}$ isochrone of D'Antona \& Mazzitelli (1997), Baraffe et al. (1998), and Chabrier et al. (2000) with the temperature scales of Schmidt-Kaler (1982) and Luhman et al. (2003b) to directly convert spectral types to masses. Relative properties (mass ratios $q$ ) for all binaries in our sample were calculated from the observed flux ratios $(\Delta K$ or $\Delta H$ ) by combining these isochrones and temperature scales with the empirical NIR colors and bolometric corrections that we compiled in Kraus \& Hillenbrand (2007b). We note that this method assumes that both components are coeval (e.g., Kraus \& Hillenbrand 2009b) and have identical extinction; the latter assumption is untested for binary systems on the scale of our newly discovered systems ( $<50 \mathrm{AU})$, and is known to fail for some wider systems (e.g., Connelley et al. 2008) including HL Tau/XZ Tau (Kenyon \& Hartmann 1995) and T Tau (Ratzka et al. 2009), as well as for systems like $\mathrm{HV}$ Tau AB-C where one component is seen only in scattered light (Duchêne et al. 2010). We also used these techniques to estimate masses for all of our sample members, which we list in Tables 1 and 5 . 

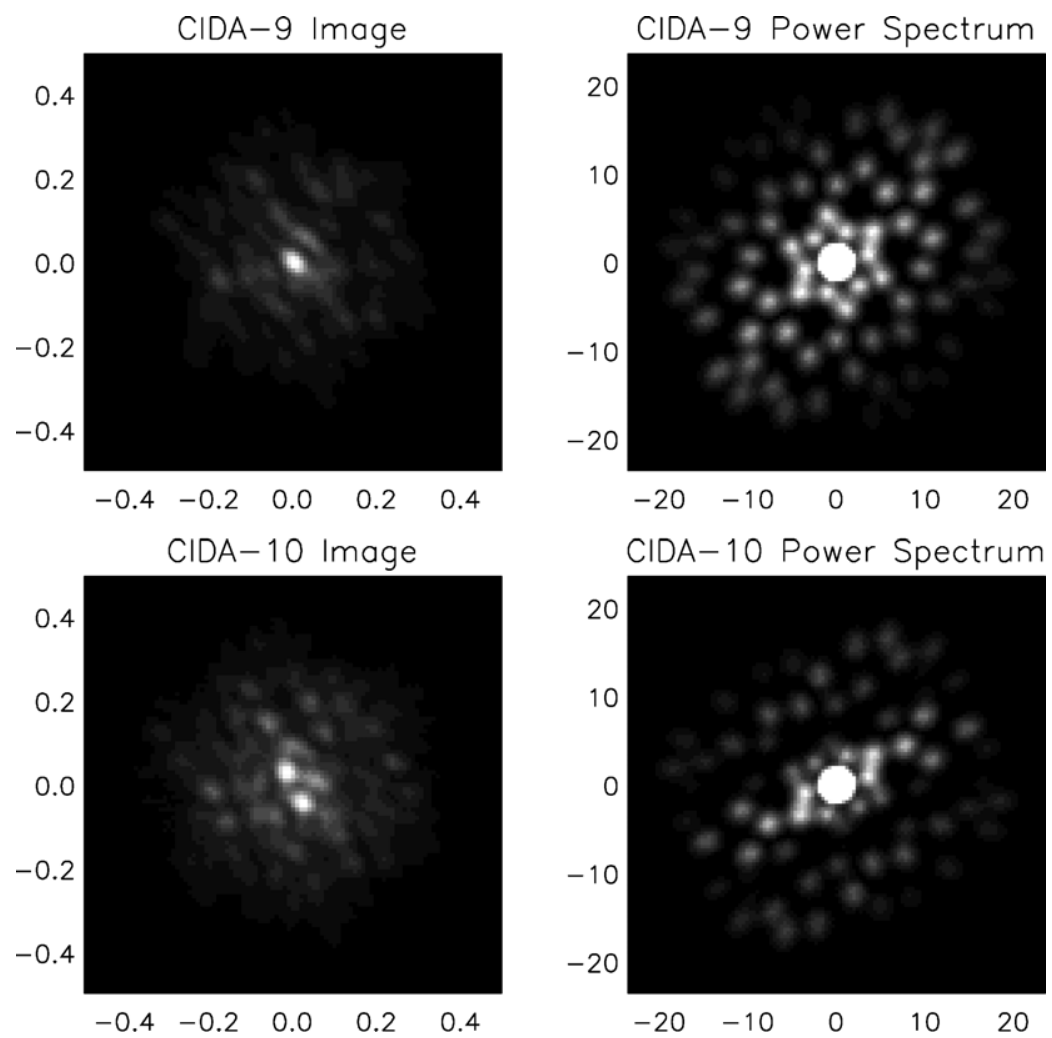

Figure 1. Interferograms (left) and power spectra (right) for the systems CIDA-9 A (no companion resolved with aperture masking) and CIDA-10 AB. Units are in arcsec (for the images) and cycles per arcsec (for the power spectra), both in on-chip coordinates.

For all binary systems, we have adopted the previously measured (unresolved) spectral type for the brightest component and inferred its properties from that spectral type. This should be a robust assumption since equal-flux binary components will have similar spectral types and significantly fainter components would not have contributed significant flux to the original discovery spectrum. We adopted a characteristic distance for all Taurus members of $145 \pm 15 \mathrm{pc}$. Recent high-precision parallax measurements with the VLBA (Loinard et al. 2007; Torres et al. 2007, 2009) and from binary orbit fitting (Boden et al. 2007) suggest that there might be a distance gradient of $165-125 \mathrm{pc}$ in the east-west direction, especially given the consistent distances of neighboring stars V773 Tau and Hubble 4 (136.2 \pm $3.7 \mathrm{pc}$ versus $132.5 \pm 0.6 \mathrm{pc}$; Boden et al. 2007; Torres et al. 2007). However, a more detailed estimate of individual distances should be postponed until the overall structure of Taurus is better sampled.

Finally, for some of our sample members, the sensitivity limits of our survey extend to the bottom of the brown dwarf mass range and could potentially encompass the top of the planetary mass range. However, mass estimates for young giant planets are completely uncalibrated and there are ongoing debates regarding their peak and typical luminosities. The models of Baraffe et al. (2003) imply that a survey sensitive to $K \sim 16$ could detect $3 M_{\text {Jup }}$ planets at the distance and age of Taurus. However, more detailed models of planet formation by Marley et al. (2007) and Fortney et al. (2008) suggest that accretion shocks can dispel much of the initial energy, leading to lower internal entropy and initial temperatures. At the typical age of Taurus members $(\sim 1-2 \mathrm{Myr})$, the luminosity of a planet could be 1-2 orders of magnitude lower than previously predicted. We cannot currently resolve this controversy, so we only note that our limits on the presence of massive planets should be considered with caution.

\section{NEW COMPANIONS AND THE MULTIPLE STELLAR POPULATION IN TAURUS-AURIGA}

Our aperture-masking observations are sensitive to near equal-flux companions at separations of $>10$ mas and can detect very faint companions $(\Delta m \sim 5.0-6.5 \mathrm{mag})$ at separations of $\gtrsim 40$ mas. The outer working angle for aperture masking, 360 mas, is set by the smallest baseline between subapertures. Companions outside this limit can still be identified, but masking observations lose sensitivity and are quickly surpassed in sensitivity by conventional AO imaging. We conservatively estimate that visual inspection of the raw interferogram images (e.g., Figure 1, left) would have revealed any binary companions with separations of $\sim 0$ '.3-2" and flux ratios $\Delta m \lesssim 2.5$, but none were found. In the vast majority of cases, this regime of parameter space has already been sampled by previous observations.

We list our newly identified binary companions and the associated detection limits for all stars in Tables 3 and 4; we found a total of 16 new companions among the 82 young stars we observed from our statistical sample. We found no additional companions to the other 10 stars that we observed in our campaign, but do not include them in our statistical analysis. We also show the binary companions and observed detection limits for our sample in Figure 2, where we plot the flux ratio $\Delta m$ as a function of projected angular separation. In addition to the companions listed, there may be more companions below our detection limit that can nonetheless be confirmed by our data. For example, some of the points used to fit the orbit of GJ 802 in Ireland et al. (2008) would have fallen below our 99.9\% confidence limit here. Most notably, the transition disk system UX Tau had a detection at 6.28 mag contrast and at a separation of 65 mas that was above the $99.9 \%$ threshold 
Table 3

Resolved Binary Companions

\begin{tabular}{|c|c|c|c|c|c|c|}
\hline Primary & Telescope & Filter & $\begin{array}{l}\text { Epoch } \\
\text { (MJD) }\end{array}$ & $\begin{array}{l}\text { Separation } \\
\text { (mas) }\end{array}$ & $\begin{array}{l}\text { P.A. } \\
(\mathrm{deg})\end{array}$ & $\begin{array}{c}\Delta m \\
(\mathrm{mag})\end{array}$ \\
\hline $2 \mathrm{M} 04080782$ & Keck & $\mathrm{Kp}$ & 54822.5 & $44.0 \pm 0.9$ & $133.1 \pm 0.8$ & $2.67 \pm 0.04$ \\
\hline Anon 1 & Keck & $\mathrm{Kp}$ & 54821.3 & $14.9 \pm 0.5$ & $144 \pm 3$ & $0.46 \pm 0.09$ \\
\hline LkCa 5 & Keck & $\mathrm{Kp}$ & 54427.4 & $48 \pm 4$ & $61 \pm 3$ & $2.89 \pm 0.16$ \\
\hline Hubble 4 & Keck & $\mathrm{Kp}$ & 54427.5 & $28.4 \pm 0.1$ & $106.07 \pm 0.13$ & $0.40 \pm 0.01$ \\
\hline LkCa 21 & Keck & $\mathrm{Kp}$ & 54428.5 & $44.4 \pm 0.1$ & $113.6 \pm 0.2$ & $0.35 \pm 0.01$ \\
\hline $\mathrm{J} 1-507$ & Keck & $\mathrm{Kp}$ & 54823.3 & $79.4 \pm 0.3$ & $19.22 \pm 0.13$ & $0.03 \pm 0.01$ \\
\hline V827 Tau & Palomar & $\mathrm{H}$ & 54434.3 & $92.9 \pm 0.3$ & $14 \pm 0.2$ & $0.58 \pm 0.01$ \\
\hline FF Tau & Keck & $\mathrm{Kp}$ & 54427.6 & $36.3 \pm 0.4$ & $356.4 \pm 0.5$ & $1.03 \pm 0.02$ \\
\hline HP Tau-G3 & Keck & $\mathrm{Kp}$ & 54427.6 & $30.5 \pm 1.5$ & $94.2 \pm 1.2$ & $1.44 \pm 0.14$ \\
\hline HV Tau & Keck & $\mathrm{Kp}$ & 54821.5 & $36.0 \pm 0.2$ & $326.6 \pm 0.3$ & $0.63 \pm 0.01$ \\
\hline $\mathrm{CoKu} \mathrm{Tau} / 4$ & Keck & $\mathrm{Kp}$ & 54427.6 & $54.1 \pm 0.3$ & $306.7 \pm 0.4$ & $0.23 \pm 0.01$ \\
\hline $2 \mathrm{M} 04414565 \mathrm{~A}$ & Keck & $\mathrm{Kp}$ & 54822.3 & $224.0 \pm 0.4$ & $87.10 \pm 0.11$ & $2.96 \pm 0.03$ \\
\hline DP Tau & Keck & $\mathrm{Kp}$ & 54428.5 & $106.7 \pm 0.8$ & $293.3 \pm 0.3$ & $0.36 \pm 0.02$ \\
\hline НBC 427 & Keck & $\mathrm{Kp}$ & 54823.5 & $32.3 \pm 0.1$ & $180.24 \pm 0.13$ & $0.87 \pm 0.01$ \\
\hline CIDA-10 & Keck & $\mathrm{Kp}$ & 54822.3 & $83.0 \pm 0.2$ & $60.47 \pm 0.22$ & $0.01 \pm 0.01$ \\
\hline CIDA-11 & Keck & $\mathrm{Kp}$ & 54822.5 & $97.2 \pm 0.2$ & $277.66 \pm 0.08$ & $0.31 \pm 0.01$ \\
\hline
\end{tabular}

Table 4

Companion Detection Limits

\begin{tabular}{|c|c|c|c|c|c|c|c|c|c|}
\hline \multirow[t]{2}{*}{ Primary } & \multirow[t]{2}{*}{ Telescope } & \multirow[t]{2}{*}{ Filter } & \multirow{2}{*}{$\begin{array}{l}\text { Epoch } \\
\text { (MJD) }\end{array}$} & \multicolumn{6}{|c|}{$\Delta m$ (mag) at $\rho=($ mas $)$} \\
\hline & & & & $10-20^{\mathrm{a}}$ & $20-40$ & $40-80$ & $80-160$ & $160-240$ & $240-320$ \\
\hline HBC 352 & Palomar & Ks & 54434.3 & 0.00 & 0.00 & 2.69 & 3.97 & 4.09 & 4.06 \\
\hline HBC 353 & Palomar & Ks & 54434.3 & 0.00 & 0.00 & 2.05 & 3.58 & 3.70 & 3.69 \\
\hline 2M04080782 & Keck & $\mathrm{Kp}$ & 54822.5 & 0.99 & 3.21 & 4.61 & 4.58 & 4.23 & 4.17 \\
\hline LkCa 1 & Keck & $\mathrm{Kp}$ & 54427.4 & 0.00 & 1.12 & 3.03 & 2.93 & 4.33 & 4.36 \\
\hline LkCa 1 & Keck & $\mathrm{Kp}$ & 54823.5 & 3.41 & 5.08 & 6.01 & 5.80 & 4.58 & 4.54 \\
\hline Anon 1 & Keck & $\mathrm{Kp}$ & 54821.3 & 1.95 & 3.82 & 5.13 & 5.07 & 5.69 & 5.74 \\
\hline FM Tau & Keck & $\mathrm{Kp}$ & 54428.6 & 0.00 & 2.19 & 3.67 & 3.49 & 4.04 & 3.99 \\
\hline FM Tau & Keck & $\mathrm{Kp}$ & 54823.3 & 1.77 & 3.77 & 5.13 & 5.04 & 4.28 & 4.24 \\
\hline FN Tau & Keck & $\mathrm{Kp}$ & 54822.5 & 1.9 & 3.84 & 5.06 & 4.87 & 3.61 & 3.57 \\
\hline CW Tau & Keck & $\mathrm{Kp}$ & 54428.4 & 1.18 & 3.37 & 4.48 & 4.37 & 4.25 & 4.24 \\
\hline
\end{tabular}

Note. ${ }^{\text {a }}$ Each column reports the detection limit (in terms of flux ratio $\Delta m$ ) for a bin with the given range of projected separations (in mas).

(This table is available in its entirety in a machine-readable form in the online journal. A portion is shown here for guidance regarding its form and content.)

by 0.03 mag. Its close proximity to the limits and potentially planetary nature suggest that we should treat it with caution until we can confirm it, so we do not yet include it in our analysis. We attempted to re-confirm the companion with deep $L^{\prime}$-band aperture masking in 2009; based on a preliminary analysis, the data were good enough to detect the candidate companion if its color were $K^{\prime}-L^{\prime}>0.5$. We are also in the process of making and analyzing follow-up observations of our binary detections. One object, $\mathrm{LkCa} 4 \mathrm{~B}$, was not detected in the followup observations. At this point, we cannot rule out either source variability or a yet unidentified systematic in data taken under poor seeing conditions, so we do not include it in our analysis either.

As we described in Sections 1 and 2, Taurus-Auriga has been the target of numerous multiplicity surveys over the past two decades. Though our results represent a significant leap forward, our newly discovered binary systems still comprise only a significant minority of all known systems in Taurus. For separations of $<4^{\prime \prime}$, previous surveys have discovered 57 additional binary companions to Taurus members with spectral types of G0-M4, some of which combine to form high-order multiple systems. Also, as we have described in past surveys,

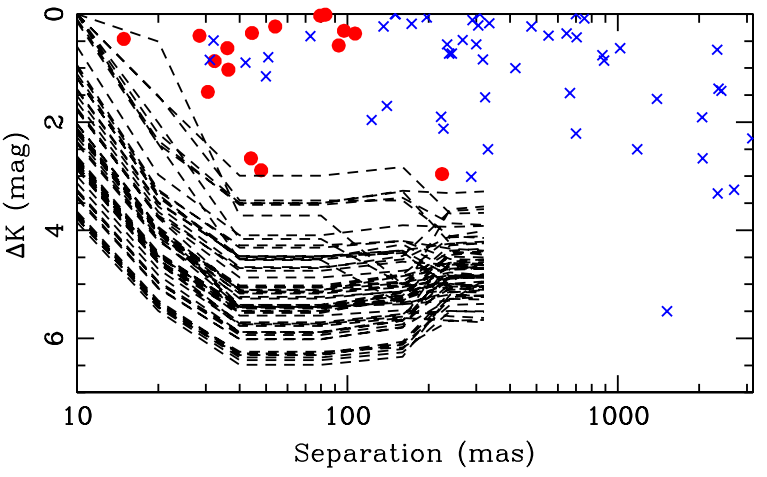

Figure 2. Detections and detection limits for our survey of Taurus-Auriga. We plot the flux ratio $\Delta m$ (in magnitudes) as a function of projected separation (in mas) for each of our newly discovered companions (red filled circles) and the known companions to our sample members (blue crosses). We also show the detection limits for all apparently single stars in our sample (black dashed lines). Most companions fall well above the detection limits, but some companions could be substellar if their low luminosity is not a result of a systematic effect (such as the presence of obscurative circumstellar material along the line of sight, as for HV Tau C or FV Tau B).

(A color version of this figure is available in the online journal.) 

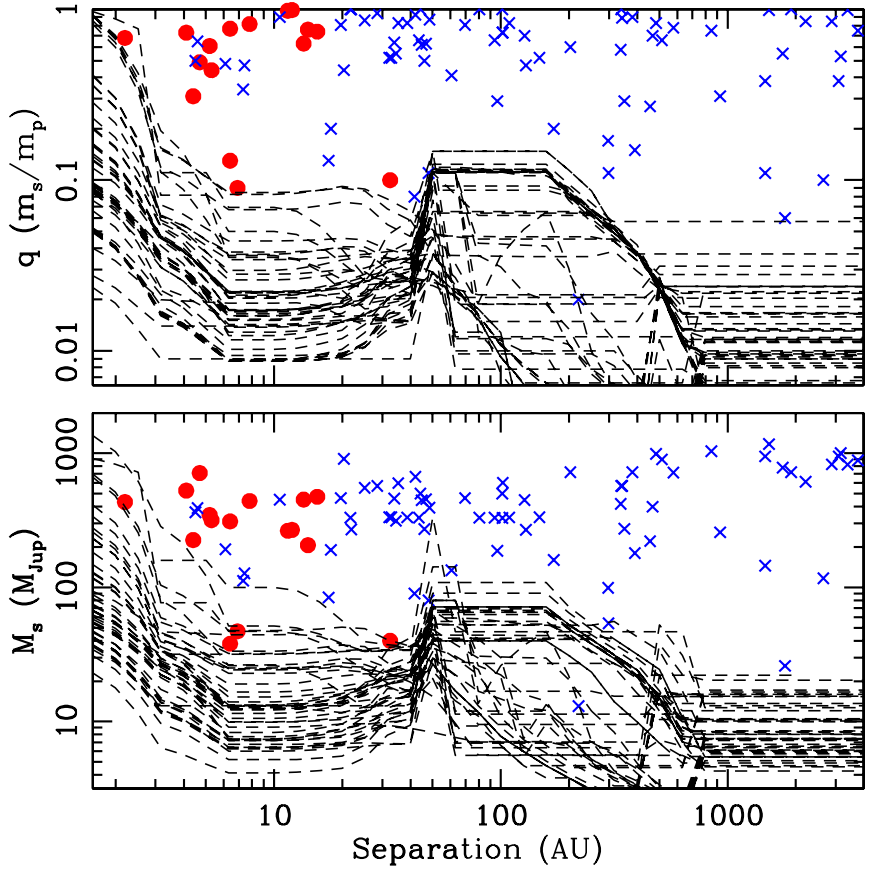

Figure 3. Detections and detection limits for our statistical sample, encompassing all of our observations as well as the detection limits adopted from the literature. The limits have been converted into mass ratios $\left(q=m_{s} / m_{p}\right)$ and companion masses (in $M_{\text {Jup }}$ ). Symbols are the same as for Figure 2. Spikes in the typical detection limits can be seen at separations of $\sim 50 \mathrm{AU}$ and $\sim 500 \mathrm{AU}$; these result from gaps where the outer working angle of one observing technique does not quite reach the inner working angle of another technique. Our Bayesian analysis (Section 5.2) naturally accounts for these narrow regions.

(A color version of this figure is available in the online journal.)

many of our observed targets are not truly independent systems but instead are bound into wide binary pairs with separations as wide as 30" (Kraus \& Hillenbrand 2008, 2009a); our statistical sample includes 17 such pairs.

In Table 5, we summarize the properties of all binary pairs that have primary stars with $M=0.25-2.5 M_{\odot}$. This sample illustrates the wide variety of possible outcomes in multiple star formation, with the most highly multiple system (V955 Tau) containing at least six components. In Table 6, we list the corresponding detection limits from our survey and from the literature for all of the apparently single stars, as well as the limits for additional companions in all of the known multiple systems. We summarize the observed properties and total detection limits for our statistical sample in Figure 3, where we plot the mass ratio and companion mass as a function of projected physical separation.

As we show in Table 5 and Figure 3, several Taurus members in our sample have companions with apparently substellar masses, including some that fall well below the stellar regime $\left(M \lesssim 50 M_{\mathrm{Jup}}\right)$. These masses were determined only from the companion's flux ratio with respect to the primary, so some could be biased by systematic effects such as circumstellar disk excesses, accretion-based stellar variability, or differential extinction. Some binary companions, such as HV Tau C, HL Tau, and V710 Tau C, are even obscured by circumstellar envelopes or edge-on disks that completely block the star along our line of sight; they can only be seen in scattered light, and thus appear underluminous by many magnitudes. However, several of these companions are very likely to be substellar since they have known spectral types (e.g., 2M04414565 B) or are found in systems with no evidence that a disk is present (e.g., LkCa 5 B,
DI Tau B; Rebull et al. 2010) or at separations of $\lesssim 50$ AU (Haro 6-37 Ab, 2M04080782 B) where binarity seems to prohibit formation of circumstellar disks (as will be shown in Kraus et al. 2011).

If we assume that this subset of companions is not biased by large systematic effects, then at least $5 / 129$ or $>3.9_{-1.2}^{+2.6} \%$ of the targets in our observed sample have a substellar companion with a separation of 5-5000 AU. This lower limit is similar to the completeness-corrected frequency of $3.2_{-2.7}^{+3.1} \%$ reported by Metchev \& Hillenbrand (2009) for a slightly narrower range of separations (28-1590 AU) and companion masses (12-72 $M_{\text {Jup }}$ ). However, the substellar companion frequency for Taurus may prove to be much higher if some of the wider companions (JH 112 Ab, JH 223 B, and StHa 34 B) are shown to be substellar or if some of the targets which were not amenable to masking observations host additional companions. We therefore suggest that the frequency could be higher by as much as a factor of $\sim 2$ over this separation range. In fact, at least one additional sample member hosts a confirmed substellar companion (DH Tau B; Itoh et al. 2005), but the companion was not included in our statistical sample since the discovery survey did not report its null detections or detection limits.

Some of our observations were sensitive to even lower masses, with a handful reaching deep into the planetary-mass regime ( 5-7 $M_{\text {Jup }}$ ). Our survey of Upper Scorpius (Kraus et al. 2008) reached the planetary-mass range for many targets, allowing us to place constraints on the properties of the exoplanet population. However, most of the Taurus targets with the best contrast limits (i.e., which were observed in periods of the best seeing) are higher-mass stars, for which the same contrast limit could detect only higher-mass companions. Only 15 targets have contrast limits deep enough to detect a $10 M_{\text {Jup }}$ planet at $10 \mathrm{AU}$, and over half have detection limits of $15 M_{\mathrm{Jup}}$ or higher, so we have not repeated that analysis.

Finally, it is noteworthy that the young binary system HBC 427 already has been identified as a single-line spectroscopic binary by Mathieu et al. (1989). The companion that we identified has a projected separation of 32 mas $(\sim 4.6 \mathrm{AU})$ and an apparent mass ratio of $\sim 0.7$ (indicating a total system mass of $\sim 1.4 M_{\odot}$ ). Massarotti et al. (2005) calculated an orbital solution with a period of $\sim 7 \mathrm{yr}$ and an eccentricity of $e \sim 0.47$; for a system mass of $\sim 1.4 M_{\odot}$, their orbit places the semimajor axis and apastron distance at $\sim 4 \mathrm{AU}$ and $\sim 6 \mathrm{AU}$, respectively. We therefore conclude that the companion we resolved is the unseen spectroscopic companion, and since the full RV curve has been determined already, this system presents an excellent prospect for a precise dynamical mass in the near future. This analysis has already been pursued by Steffen et al. (2001) using astrometry from the Hubble Space Telescope Fine Guidance Sensors, but an extrapolation of their orbital solution yields an inconsistent prediction for the companion position in our observations, so an updated solution seems to be required.

\section{THE MASS-DEPENDENT PROPERTIES OF YOUNG MULTIPLE SYSTEMS}

Our new, significantly more complete binary census of Taurus offers a unique opportunity to study the primordial outcome of multiple star formation. In the following subsections, we approach this question from several angles. First, we construct histograms of the separation distribution and mass-ratio distribution in order to determine what functional forms seem most appropriate for their description. Next, we use Bayesian analysis techniques to estimate the relevant scale parameters for 
Table 5

Derived Properties for Class II/III Binaries in Taurus

\begin{tabular}{|c|c|c|c|c|c|c|c|}
\hline $\begin{array}{l}\text { Primary } \\
\text { Name }\end{array}$ & $\begin{array}{l}\text { Secondary } \\
\text { Name }\end{array}$ & $\begin{array}{c}M_{\text {prim }}{ }^{\mathrm{a}} \\
\left(M_{\odot}\right)\end{array}$ & $\begin{array}{l}M_{\mathrm{sec}^{\mathrm{a}}} \\
\left(M_{\odot}\right)\end{array}$ & $\begin{array}{c}q^{\mathrm{b}} \\
\left(M_{s} / M_{p}\right)\end{array}$ & $\begin{array}{l}\mathrm{Sep}^{\mathrm{c}} \\
(\mathrm{AU})\end{array}$ & $\begin{array}{c}q \\
\text { Type }^{\mathrm{b}}\end{array}$ & References \\
\hline 2M04080782 A & 2M04080782 B & 0.30 & $(0.04)$ & 0.13 & 6.4 & $\Delta K$ & 1 \\
\hline Anon $1 \mathrm{~A}$ & Anon 1 B & 0.64 & $(0.43)$ & 0.68 & 2.2 & $\Delta K$ & 1 \\
\hline MHO 2 A & MHO 2 B & 0.33 & $(0.11)$ & 0.34 & 7.3 & $\Delta K$ & 2 \\
\hline MHO $3 \mathrm{~A}$ & MHO 3 B & 0.72 & $(0.36)$ & 0.50 & 4.5 & $\Delta K$ & 2 \\
\hline $\mathrm{LkCa} 3 \mathrm{~A}$ & LkCa 3 B & 0.57 & $(0.46)$ & 0.81 & 69 & $\Delta K$ & 3 \\
\hline FO Tau A & FO Tau B & 0.33 & $(0.33)$ & 1.00 & 22 & $\Delta K$ & 3 \\
\hline LkCa 5 A & LkCa 5 B & 0.50 & $(0.05)$ & 0.09 & 6.9 & $\Delta K$ & 1 \\
\hline V410 Tau A & V410 Tau B & 0.94 & $(0.19)$ & 0.20 & 17.8 & $\Delta K$ & 4 \\
\hline V410 Tau AB & V410 Tau C & 1.13 & $(0.09)$ & 0.08 & 42 & SpT-M, $\Delta K$ & 4 \\
\hline DD Tau A & DD Tau B & 0.33 & 0.33 & 1.00 & 80 & SpT-M & 3 \\
\hline CZ Tau A & CZ Tau B & 0.54 & $(0.27)$ & 0.50 & 46 & $\Delta K$ & 3 \\
\hline V410 X-ray $7 \mathrm{~A}$ & V410 X-ray 7 B & 0.60 & $(0.39)$ & 0.65 & 4.6 & $\Delta K$ & 2 \\
\hline Hubble 4 A & Hubble 4 B & 0.72 & $(0.53)$ & 0.73 & 4.1 & $\Delta K$ & 1 \\
\hline FQ Tau A & FQ Tau B & 0.40 & 0.33 & 0.83 & 109 & SpT-M & 3 \\
\hline $\mathrm{LkCa} 7 \mathrm{~A}$ & $\mathrm{LkCa} 7 \mathrm{~B}$ & 0.64 & 0.33 & 0.52 & 148 & SpT-M & 3 \\
\hline FS Tau A & FS Tau B & 0.64 & 0.33 & 0.52 & 33 & SpT-M & 3 \\
\hline FS Tau AB & Haro 6-5B & 0.97 & 0.82 & 0.85 & 2900 & SpT-M & 5 \\
\hline $\mathrm{LkCa} 21 \mathrm{~A}$ & $\mathrm{LkCa} 21 \mathrm{~B}$ & 0.4 & $(0.31)$ & 0.77 & 6.4 & $\Delta K$ & 1 \\
\hline J1-4872 Aa & $\mathrm{J} 1-4872 \mathrm{Ab}$ & 0.64 & $(0.55)$ & 0.86 & 25 & $\Delta K$ & 6 \\
\hline J1-4872 Aab & $\mathrm{J} 1-4872 \mathrm{Bab}$ & 1.19 & 0.99 & 0.83 & 480 & SpT-M, $\Delta K$ & 6 \\
\hline FV Tau A & FV Tau B & 0.82 & $(0.60)$ & 0.73 & 102 & $\Delta K$ & 3 \\
\hline FV Tau /c A & FV Tau /c B & 0.45 & 0.33 & 0.73 & 102 & SpT-M & 3 \\
\hline FV Tau AB & FV Tau /c AB & 1.42 & 0.78 & 0.55 & 1750 & SpT-M, $\Delta K$ & 6 \\
\hline DF Tau A & DF Tau B & 0.50 & 0.45 & 0.90 & 10.6 & SpT-M & 7 \\
\hline $\mathrm{J} 1-507 \mathrm{~A}$ & J1-507 B & 0.27 & $(0.26)$ & 0.98 & 11.5 & $\Delta K$ & 1 \\
\hline FW Tau A & FW Tau B & 0.27 & $(0.27)$ & 1.00 & 22 & $\Delta K$ & 8 \\
\hline DI Tau A & DI Tau B & 0.64 & $(0.08)$ & 0.13 & 17.4 & $\Delta K$ & 9 \\
\hline DI Tau AB & DH Tau AB & 0.72 & 0.61 & 0.85 & 2200 & SpT-M, $\Delta K$ & 5 \\
\hline UXTau A & UX Tau C & 1.20 & 0.18 & 0.15 & 390 & SpT-M & 6 \\
\hline UX Tau Ba & UX Tau Bb & 0.57 & $(0.46)$ & 0.81 & 19.7 & $\Delta K$ & 6 \\
\hline UXTau AC & UX Tau Bab & 1.38 & 1.03 & 0.75 & 850 & SpT-M, $\Delta K$ & 10 \\
\hline FX Tau A & FX Tau B & 0.57 & 0.27 & 0.47 & 129 & SpT-M & 3 \\
\hline DK Tau A & DK Tau B & 0.64 & 0.57 & 0.89 & 340 & SpT-M & 6 \\
\hline ZZ Tau A & ZZ Tau B & 0.40 & $(0.19)$ & 0.48 & 6.1 & $\Delta K$ & 11 \\
\hline V927 Tau A & V927 Tau B & 0.40 & 0.33 & 0.83 & 39 & SpT-M & 3 \\
\hline XZ Tau A & XZ Tau B & 0.50 & 0.33 & 0.66 & 43 & SpT-M & 3 \\
\hline XZ Tau AB & HL Tau & 0.83 & 0.82 & 0.99 & 3400 & SpT-M & 5 \\
\hline HK Tau A & HK Tau B & 0.57 & 0.57 & 1.00 & 340 & SpT-M & 3 \\
\hline V710 Tau A & V710 Tau B & 0.57 & 0.40 & 0.70 & 470 & SpT-M & 6 \\
\hline V710 Tau AB & V710 Tau C & 0.97 & 0.40 & 0.41 & 4100 & SpT-M & 5 \\
\hline V827 Tau A & V827 Tau B & 0.72 & $(0.45)$ & 0.63 & 13.5 & $\Delta H$ & 1 \\
\hline V928 Tau A & V928 Tau B & 0.60 & $(0.57)$ & 0.95 & 29 & $\Delta K$ & 3 \\
\hline V928 Tau AB & CFHT-Tau-7 & 1.17 & 0.12 & 0.10 & 2600 & SpT-M, $\Delta K$ & 5 \\
\hline GG Tau Aa & GG Tau Ab & 0.72 & 0.60 & 0.83 & 35 & SpT-M & 3 \\
\hline GG Tau Aab & GG Tau Bab & 1.32 & 0.14 & 0.11 & 1460 & SpT-M & 6 \\
\hline UZ Tau Aab & UZ Tau Bab & 1.36 & 0.90 & 0.66 & 510 & DynM, SpT-M & 3 \\
\hline JH $112 \mathrm{Aa}$ & $\mathrm{JH} 112 \mathrm{Ab}$ & 0.82 & $(0.01)$ & 0.02 & 220 & $\Delta K$ & 2 \\
\hline JH 112 Aab & JH112 Bab & 0.83 & 0.26 & 0.31 & 930 & SpT-M, $\Delta K$ & 2 \\
\hline V807 Tau A & V807 Tau Bab & 0.72 & 1.17 & 0.62 & 45 & DynM, SpT-M & 3 \\
\hline GH Tau A & GH Tau B & 0.50 & 0.50 & 1.00 & 44 & SpT-M & 3 \\
\hline V807Tau ABab & GH Tau AB & 1.89 & 1.00 & 0.53 & 3200 & DynM, SpT-M & 5 \\
\hline GK Tau & GI Tau & 0.72 & 0.72 & 1.00 & 1910 & SpT-M & 5 \\
\hline IS Tau A & IS Tau B & 0.64 & 0.33 & 0.52 & 32 & SpT-M & 3 \\
\hline HN Tau A & HN Tau B & 0.82 & 0.22 & 0.27 & 460 & SpT-M & 6 \\
\hline IT Tau A & IT Tau B & 0.94 & 0.27 & 0.29 & 350 & SpT-M & 6 \\
\hline J2-2041 A & $\mathrm{J} 2-2041 \mathrm{~B}$ & 0.33 & $(0.13)$ & 0.41 & 61 & $\Delta K$ & 2 \\
\hline HBC 407 A & HBC 407 B & 2.08 & $(0.91)$ & 0.44 & 20 & $\Delta I$ & 12 \\
\hline FF Tau A & FF Tau B & 0.72 & $(0.32)$ & 0.44 & 5.3 & $\Delta K$ & 1 \\
\hline HBC 412 A & HBC 412 B & 0.50 & $(0.50)$ & 1.00 & 102 & $\Delta K$ & 13 \\
\hline $\mathrm{CoKu} \mathrm{Tau} / 3 \mathrm{~A}$ & CoKu Tau/3 B & 0.57 & $(0.10)$ & 0.17 & 300 & $\Delta K$ & 3 \\
\hline HP Tau G3 A & HP Tau G3 B & 0.72 & $(0.23)$ & 0.31 & 4.4 & $\Delta K$ & 1 \\
\hline HP Tau G2 & HP Tau & 2.49 & 0.94 & 0.38 & 3100 & SpT-M & 5 \\
\hline HP Tau G2 & HP Tau G3 AB & 2.49 & 0.95 & 0.38 & 1460 & SpT-M, $\Delta K$ & 5 \\
\hline Haro 6-28 A & Haro 6-28 B & 0.50 & 0.33 & 0.66 & 94 & SpT-M & 3 \\
\hline HV Tau A & HV Tau B & 0.57 & $(0.35)$ & 0.61 & 5.2 & $\Delta K$ & 1 \\
\hline
\end{tabular}


Table 5

(Continued)

\begin{tabular}{|c|c|c|c|c|c|c|c|}
\hline $\begin{array}{l}\text { Primary } \\
\text { Name }\end{array}$ & $\begin{array}{l}\text { Secondary } \\
\text { Name }\end{array}$ & $\begin{array}{c}M_{\text {prim }}{ }^{\mathrm{a}} \\
\left(M_{\odot}\right)\end{array}$ & $\begin{array}{l}M_{\mathrm{sec}^{\mathrm{a}}} \\
\left(M_{\odot}\right) \\
\end{array}$ & $\begin{array}{c}q^{\mathrm{b}} \\
\left(M_{s} / M_{p}\right) \\
\end{array}$ & $\begin{array}{l}\text { Sep } \\
(\mathrm{AU})\end{array}$ & $\begin{array}{c}q \\
\text { Type }^{\mathrm{b}}\end{array}$ & References \\
\hline HV Tau AB & HV Tau C & 0.92 & 0.72 & 0.78 & 580 & SpT-M, $\Delta K$ & 14 \\
\hline VY Tau A & VY Tau B & 0.64 & $(0.19)$ & 0.29 & 96 & $\Delta K$ & 3 \\
\hline GN Tau A & GN Tau B & 0.45 & $(0.39)$ & 0.87 & 49 & $\Delta K$ & 3 \\
\hline JH 223 A & JH 223 B & 0.50 & $(0.05)$ & 0.11 & 300 & $\Delta K$ & 5 \\
\hline IW Tau A & IW Tau B & 0.72 & $(0.67)$ & 0.93 & 42 & $\Delta K$ & 3 \\
\hline CoKu Tau/4 A & CoKu Tau/4 B & 0.54 & $(0.44)$ & 0.82 & 7.8 & $\Delta K$ & 15 \\
\hline 2M04414565 Aa & $2 \mathrm{M} 04414565 \mathrm{Ab}$ & 0.40 & $(0.04)$ & 0.10 & 32 & $\Delta K$ & 1 \\
\hline 2M04414565 Aab & 2M04414565 B & 0.44 & 0.027 & 0.06 & 1790 & SpT-M, $\Delta K$ & 5 \\
\hline LkHa 332 G2 A & LkHa 332 G2 B & 0.72 & $(0.46)$ & 0.64 & 34 & $\Delta K$ & 3 \\
\hline V955 Tau A & V955 Tau B & 0.72 & 0.45 & 0.63 & 47 & SpT-M & 3 \\
\hline LkHa 332 G1 A & LkHa 332 G1 B & 0.57 & $(0.31)$ & 0.55 & 35 & $\Delta K$ & 3 \\
\hline LkHa 332 G2 AB & V955 Tau AB & 1.18 & 1.17 & 0.99 & 1520 & SpT-M, $\Delta K$ & 5 \\
\hline LkHa 332 G2 AB & LkHa 332 G1 AB & 1.18 & 0.88 & 0.75 & 3800 & SpT-M, $\Delta K$ & 5 \\
\hline DP Tau A & DP Tau B & 0.64 & $(0.47)$ & 0.74 & 15.5 & $\Delta K$ & 1 \\
\hline RX J0446.7+2459 A & RX J0446.7+2459 B & 0.27 & $(0.13)$ & 0.47 & 7.4 & $\Delta K$ & 2 \\
\hline Haro 6-37 Aa & Haro 6-37 Ab & 0.72 & $(0.08)$ & 0.11 & 48 & $\Delta K$ & 16 \\
\hline Haro 6-37 Aab & Haro 6-37 B & 0.80 & 0.72 & 0.90 & 380 & SpT-M, $\Delta K$ & 3 \\
\hline UY Aur A & UY Aur B & 0.64 & 0.45 & 0.70 & 127 & SpT-M & 3 \\
\hline StHa $34 \mathrm{Aab}$ & StHa 34 B & 0.80 & $(0.08)$ & 0.20 & 171 & $\Delta K$ & 2 \\
\hline HBC 427 A & HBC $427 \mathrm{~B}$ & 1.45 & 0.81 & 0.49 & 4.7 & DynMass & 1 \\
\hline CIDA-9 A & CIDA-9 B & 0.72 & $(0.42)$ & 0.58 & 340 & $\Delta K$ & 5 \\
\hline CIDA-10 A & CIDA-10 B & 0.27 & $(0.27)$ & 0.99 & 12.0 & $\Delta K$ & 1 \\
\hline CIDA-11 A & CIDA-11 B & 0.27 & $(0.21)$ & 0.76 & 14.1 & $\Delta K$ & 1 \\
\hline RW Aur A & RW Aur B & 1.20 & 0.72 & 0.60 & 200 & SpT-M & 3 \\
\hline
\end{tabular}

Notes.

${ }^{a}$ The primary mass was determined from its corresponding spectral type, as described in Section 3.3. A majority of the secondary masses were determined from the primary mass and the calculated mass ratio (quantities in parentheses), but many were directly calculated from measured spectral type of the secondary component. The uncertainties are likely to be $\sim 20 \%$ and are set by systematic uncertainties from the models and the highly uncertain ages of Taurus members.

${ }^{\mathrm{b}}$ As we describe in Section 3.3, the mass ratio of each system was estimated from the mass-spectral-type relation described in the text (SpT-M), a dynamical mass measurement (DynM), or from the flux ratio $(\Delta I, \Delta H$, or $\Delta K)$. For some hierarchical multiple systems, several techniques were required to estimate all component masses. The uncertainties are likely to be $\sigma_{q} \sim 0.1$, but could be much larger if there are edge-on disks or unresolved components that have not been identified.

${ }^{\mathrm{c}}$ As we discuss in Section 3.3, projected physical separations are determined from the projected angular separations and the mean distance to Taurus. Projected physical separations are uncertain by $\sim 15 \%$ due to the finite spread of distances to Taurus members $(\sim 20 \mathrm{pc})$.

References. (1) This work; (2) R. White et al. 2011, in preparation; (3) White \& Ghez 2001; (4) Ghez et al. 1997; (5) Kraus \& Hillenbrand 2007a; (6) Correia et al. 2006; (7) Chen et al. 1990; (8) Woitas et al. 2001; (9) Ghez et al. 1993; (10) Duchêne et al. 1999; (11) Simon et al. 1995, 1996; (12) Sartoretti et al. 1998; (13) Leinert et al. 1993; (14) Woitas \& Leinert 1998; (15) Ireland \& Kraus 2008; (16) Richichi et al. 1998.

those functional forms. Finally, we address the supposed ubiquity of multiple star formation by directly counting the number of apparently single stars in Taurus-Auriga.

\subsection{Observed Distributions}

The current paradigm for field binary properties was established by DM91, who conducted a volume-limited multiplicity survey of solar-type stars with spectral types F7-G9. They found a separation distribution which is apparently unimodal and lognormal, with a mean semimajor axis of $\sim 30 \mathrm{AU}$ and a standard deviation of $\sim 1$ dex. They also found a mass-ratio distribution that is peaked at low masses $(q \sim 0.3)$ and has few similar-mass companions, though their survey was not sensitive to most substellar companions and relied on significant completeness corrections for low-mass stellar companions. Finally, they found that $\sim 60 \%$ of solar-type stars have at least one binary companion. The frequency and properties of binary systems appear to depend on their mass (e.g., FM92; Bouy et al. 2003; Burgasser et al. 2003; Close et al. 2003), but the mass range of DM91 is well matched to the median mass for the upper half of our sample.
Subsequent surveys of young stars have not observed the same features as in DM91, especially for less dynamically evolved populations. Our survey of wide multiplicity in Taurus and Upper Scorpius suggests that the separation distribution for solar-type stars is actually log-flat, with more wide binary companions than are seen in the field (Kraus \& Hillenbrand 2009a). We also found in our aperture-masking survey of Upper Scorpius (Kraus et al. 2008) that the mass-ratio distribution of solar-type binaries might be much less biased toward low-mass companions, with the most likely distribution being linearly flat such that all companion masses are equally probable. Most surveys of young stars in these regions (e.g., Ghez et al. 1993; Leinert et al. 1993; Simon et al. 1995; Kouwenhoven et al. 2007; Kraus et al. 2008) find a significantly higher binary frequency than in the field, such that the binary frequency of solar-type stars in Taurus might approach $100 \%$.

These discrepancies between the field and young star-forming regions show that we cannot assume that the binary properties in our sample match those in the field, so we begin our analysis with the simplest non-parametric analysis: plotting histograms of the binary properties. To this end, we plot the separation 


\begin{tabular}{|c|c|c|c|c|c|c|c|c|c|c|c|c|c|c|c|c|c|c|c|c|}
\hline \multirow[t]{2}{*}{ Primary } & \multirow{2}{*}{$\begin{array}{l}\text { Mass } \\
\left(M_{\odot}\right)\end{array}$} & \multicolumn{18}{|c|}{$q_{\lim }\left(m_{s} / m_{p}\right)$ at $\rho=(\mathrm{mas})$} & \multirow[t]{2}{*}{ References } \\
\hline & & $13.8^{\mathrm{a}}$ & 17.3 & 21.8 & 27.5 & 34.6 & 43.5 & 54.8 & 69.0 & 86.8 & 138 & 218 & 346 & 690 & 1376 & 2746 & 5478 & 10930 & 27455 & \\
\hline 2M04080782 A & 0.30 & 0.282 & 0.177 & 0.093 & 0.075 & 0.053 & 0.036 & 0.036 & 0.036 & 0.036 & 1.000 & 1.000 & 1.000 & 1.000 & 1.000 & 1.000 & 1.000 & 1.000 & 1.000 & 1 \\
\hline $2 \mathrm{M} 04080782 \mathrm{AB}$ & 0.34 & 1.000 & 1.000 & 1.000 & 1.000 & 1.000 & 1.000 & 1.000 & 1.000 & 1.000 & 0.031 & 0.036 & 0.125 & 0.125 & 0.125 & 0.102 & 0.102 & 0.102 & 0.102 & 1 \\
\hline LkCa 1 & 0.27 & 0.066 & 0.042 & 0.024 & 0.021 & 0.018 & 0.015 & 0.015 & 0.015 & 0.015 & 0.017 & 0.029 & 0.148 & 0.148 & 0.115 & 0.037 & 0.018 & 0.018 & 0.018 & 1 \\
\hline Anon 1 A & 0.64 & 0.131 & 0.087 & 0.051 & 0.042 & 0.030 & 0.020 & 1.000 & 1.000 & 1.000 & 1.000 & 1.000 & 1.000 & 1.000 & 1.000 & 1.000 & 1.000 & 1.000 & 1.000 & 1,2 \\
\hline Anon $1 \mathrm{AB}$ & 1.07 & 1.000 & 1.000 & 1.000 & 1.000 & 1.000 & 1.000 & 0.012 & 0.012 & 0.012 & 0.013 & 0.009 & 0.067 & 0.009 & 0.009 & 0.009 & 0.006 & 0.006 & 0.006 & 1,2 \\
\hline FM Tau & 0.64 & 0.157 & 0.099 & 0.053 & 0.043 & 0.030 & 0.020 & 0.020 & 0.020 & 0.021 & 0.022 & 0.037 & 0.111 & 0.111 & 0.082 & 0.038 & 0.016 & 0.016 & 0.016 & 1 \\
\hline CW Tau & 0.94 & 0.266 & 0.149 & 0.058 & 0.051 & 0.041 & 0.034 & 0.034 & 0.034 & 0.034 & 0.036 & 0.038 & 0.047 & 0.047 & 0.047 & 0.034 & 0.006 & 0.006 & 0.006 & 1,3 \\
\hline MHO-2 A & 0.33 & 1.000 & 1.000 & 1.000 & 1.000 & 1.000 & 0.175 & 0.143 & 0.133 & 0.121 & 0.104 & 1.000 & 1.000 & 1.000 & 1.000 & 1.000 & 1.000 & 1.000 & 1.000 & 4,5 \\
\hline MHO-2 AB & 0.45 & 1.000 & 1.000 & 1.000 & 1.000 & 1.000 & 1.000 & 1.000 & 1.000 & 1.000 & 1.000 & 0.066 & 0.023 & 0.011 & 0.008 & 0.008 & 0.008 & 0.008 & 1.000 & 4,5 \\
\hline MHO-2 AB+1 & 0.90 & 1.000 & 1.000 & 1.000 & 1.000 & 1.000 & 1.000 & 1.000 & 1.000 & 1.000 & 1.000 & 1.000 & 1.000 & 1.000 & 1.000 & 1.000 & 1.000 & 1.000 & 0.006 & 4,5 \\
\hline
\end{tabular}

Notes. All observation limits include our wide binary results from Kraus \& Hillenbrand (2007a, 2009a).

${ }^{a}$ Each column reports the detection limit (in terms of companion mass ratio) for a bin with the given projected separation (in mas).

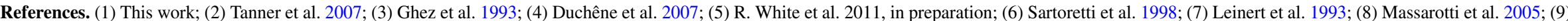
Simon et al. 1995; (10) Konopacky et al. 2007; (11) Correia et al. 2006; (12) Simon et al. 1999; (13) Richichi et al. 1998.

(This table is available in its entirety in a machine-readable form in the online journal. A portion is shown here for guidance regarding its form and content.) 


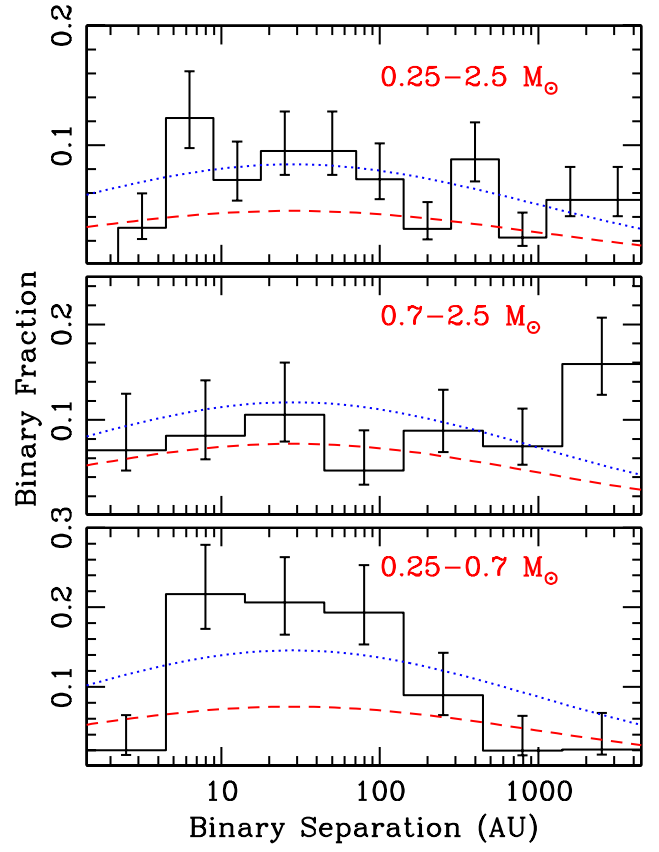

Figure 4. Separation distribution for our full sample (top) and for two subsets of primary mass. For each bin of primary mass and projected separation, we plot the companion fraction for all stars which were surveyed to a sensitivity of $q \sim$ 0.1 or better. The red dashed line denotes the separation distribution observed by DM91 as normalized to their companion fraction (39\% in this separation range), while the blue dotted line shows the same separation distribution renormalized to match the total companion fraction of our sample in that mass range (73\% for all stars, $62 \%$ for the high-mass subsample, and $77 \%$ for the low-mass subsample).

(A color version of this figure is available in the online journal.)

distribution for a range of mass ratios where our survey is nearly complete, then we plot the mass-ratio distribution for a range of separations where our survey is nearly complete. We must implicitly assume that the separation distribution and mass-ratio distribution are not correlated, but our surveys of multiplicity at small and large separations (e.g., Kraus et al. 2008 versus Kraus \& Hillenbrand 2009a) show no such correlation in other samples of similar size, so this assumption should be robust for our new Taurus sample.

In Figure 4, we show the observed separation distribution for our full sample and for two subsets of primary masses. Each bin of mass and separation represents the frequency of companions among all sample members for which we could have detected binary companions with mass ratios of $q \sim 0.1$, so the number counts vary between bins and are generally higher at larger separations (where it is easier to achieve deep detection limits). We also treat close binary pairs as a single combined mass for the purposes of tertiary companion detection, so many of the close binaries from the low-mass subsample range are represented in the wide-separation bins of the high-mass subsample. In each case, we also plot the separation distribution observed by DM91 for both their total binary frequency $(\sim 39 \%)$ and our observed total binary frequency $(63 \%-76 \%)$ across this separation range.

The overall mass-dependent trends match our expectations from previous surveys of young stars (Kraus et al. 2008; Kraus \& Hillenbrand 2009a). The separation distribution for approximately solar-mass stars $\left(0.7-2.5 M_{\odot}\right)$ appears log-flat over separations of 5-5000 AU, similar to our results for Upper Scorpius (K08; Kraus \& Hillenbrand 2009a). For lower-mass stars $\left(0.25-0.70 M_{\odot}\right)$, wide $(\gtrsim 200 \mathrm{AU})$ binary companions are

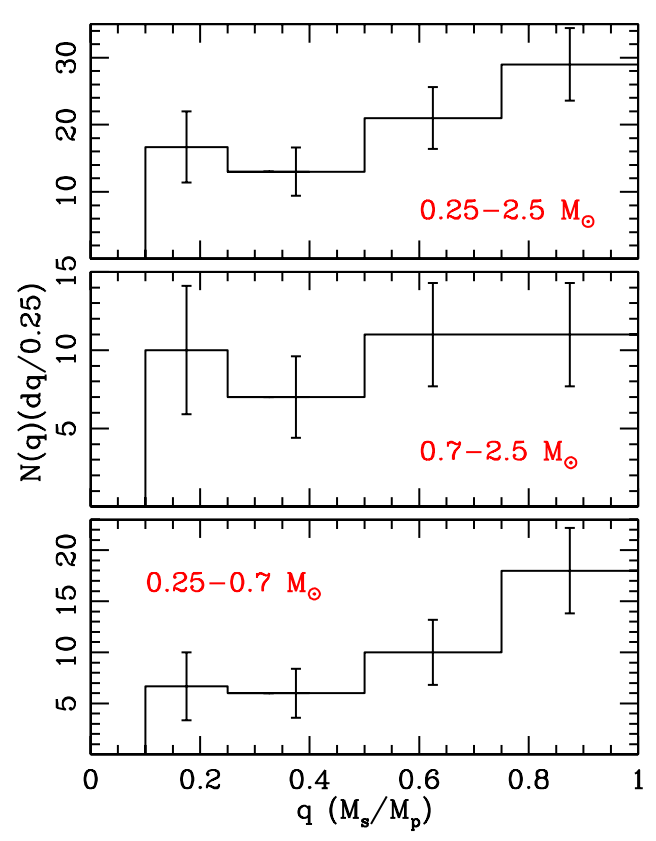

Figure 5. Mass-ratio distribution for our full sample (top) and for two subsets of primary mass. For each bin of primary mass and mass ratio, we plot the number of binary companions among all systems with projected separation $>50$ mas. As we describe in the text, we must consider number counts instead of frequencies because systems with multiple components have a different "primary mass" for the close and wide companion, rather than a single definition that spans all separations. The low- $q$ bin only extends to $q=0.1$, so we have cast the $y$-axis as the number of companions per $\Delta q=0.25$, then multiplied the number of observed companions with $0.10<q<0.25$ by a factor of $5 / 3$ in order to match this definition.

(A color version of this figure is available in the online journal.)

less common, though binary companions at smaller separations remain common. It is unclear whether the separation distribution is better modeled as a log-normal function (as for the field) where the mean and standard deviation decline with mass, or as a log-flat function with a mass-dependent outer cutoff. As we describe in the next subsection, modeling the function as log-flat leads to trivial conclusions, so we will emphasize the log-normal distribution in our subsequent analysis.

In Figure 5, we show the mass-ratio distribution for all binary companions with projected separations of $>50$ mas ( $\gtrsim 15 \mathrm{AU})$ and mass ratios $>0.1$, again for our entire sample and for two ranges of primary mass. Each bin of mass and mass ratio represents the number of companions among all of the sample members for which we could have detection binary companions with mass ratios of $q \sim 0.1$ at separations of $\sim 100$ mas. We cannot present a frequency for each bin, unlike for Figure 4, because the effective "primary mass" changes as a function of separation for systems with multiple components. Any attempt to correct for including partial separation ranges would require untested assumptions about the separation distribution, so we prefer to compromise by dealing with number counts instead of frequencies, then addressing the more comprehensive population statistics in our Bayesian analysis.

The full mass-ratio distribution is close to flat, but with a moderate excess of similar-mass companions. This distribution is a stark contrast to the results of DM91, who found few similarmass companions. However, it is much more consistent with previous surveys of young stars, which found a distribution that was close to linearly flat for solar-type stars and an increasing tendency for similar-mass companions at $\lesssim 0.3 M_{\odot}$. The 
mass-ratio distribution for $0.25-0.7 M_{\odot}$ stars has more similarmass companions than low-mass companions, but as we will show more clearly in the next subsections, the difference is only marginally significant. We will therefore follow the lead of previous Bayesian analysis implementations and will treat the mass-ratio distribution as a power law with an unconstrained exponent.

\subsection{Bayesian Analysis}

Binary population statistics are traditionally presented in terms of histograms of companion frequency versus separation or mass ratio, where the data are presented only for a range where the survey is complete (e.g., Figures 4 and 5). The analytic form of the preferred model is then fit to these histograms in order to infer the population properties. As we showed in the previous subsection, this non-parametric approach is required in cases where the functional forms of the parameter distributions are unknown. However, once a functional form can be prescribed, then a better solution for working with heterogeneous data is to adopt a Bayesian approach, where the scale parameters of the model are assigned a prior PDF and that PDF is modified by each observation. This method exploits Bayes' theorem:

$$
P(\theta \mid O) \propto P(O \mid \theta) P(\theta),
$$

where $\theta$ represents the "model" (a set of scale parameters describing the functional form), $O$ represents the observation, $P(\theta \mid O)$ is the posterior PDF for the model (as a function of its parameters) given the data, $P(O \mid \theta)$ is the probability of obtaining an observation as a function of the model parameters, and $P(\theta)$ is the prior PDF for the model (again, as a function of its parameters). In cases with multiple observations, the posterior function for one observation is then used as the prior function for the next observation.

Bayesian analysis techniques offer several compelling advantages over traditional techniques that produce histograms and fit probability density functions. The most notable distinction is that Bayesian analysis optimally exploits the available data, while implicitly avoiding any need for completeness corrections. Traditional histogram analysis requires the identification of a "complete" regime of parameter space where all observations are sensitive to the detection of companions; in some cases, this regime can be expanded by using a completeness correction for regimes of partial sensitivity. In contrast, our formulation of Bayesian analysis optimally exploits each observation by drawing value from regimes where a companion could be detected, but ignoring regimes where one could not. Bayesian analysis also avoids the uncertainties of binning, which can be significant if there are only enough observations to justify a small number of bins (e.g., Figure 5). Finally, Bayesian analysis provides a more direct measurement of physically meaningful parameters. Histograms measure the PDF of the population, and then that PDF must be fit with distributions in order to estimate the parameters that describe that population (such as the mean separation or the slope of the mass-ratio distribution). In contrast, Bayesian analysis directly yields the PDF for those parameters, bypassing the intermediate step. As we show below, this advantage can be helpful not just in showing a study's measurements of population properties, but also honestly presenting the limits on those measurements and the extent of ignorance. However, we must acknowledge a significant caveat. Bayesian analysis is only meaningful for assumed functional forms of a population, and as we showed above, histograms must be inspected first to determine whether a given functional form appears valid.
Allen (2007, hereafter A07) developed the relevant techniques for applying Bayesian statistics to multiple star populations, and we used his approach in our recent survey of multiplicity in the VLM regime (A. Kraus \& L. A. Hillenbrand 2011, in preparation). This method describes the PDF for the binary population in terms of a companion frequency $F$, a power-law mass-ratio distribution with exponent $\gamma$, and a log-normal separation distribution with mean $\overline{\log (\rho)}$ and standard deviation $\sigma_{\log (\rho)}$. Each of these parameters is given a prior, and then the observations modify this prior to yield the posterior PDF that carries our new constraints on the population. However, rather than using the conventional Bayesian approach where each observation serially modifies the prior, this method instead compiles a single "window function" $N_{\text {obs }}(q, \log (\rho))$ that describes the number of observations which are sensitive to discrete bins of separation $\log (\rho)$ and mass ratio $q$, plus a corresponding "companion function" $N_{\text {comp }}(q, \log (\rho))$ that describes the number of companions detected in each of those bins. The net effect is to treat each bin of parameter space $(\Delta q, \Delta \log (\rho))$ as an observation, then iterate through all bins so that they serially modify the prior PDF to yield a posterior PDF.

Given this assumed functional form that describes the population, for each set of model parameters the expected frequency of companions in a bin $(\Delta q, \Delta \log (\rho))$ is given by a probability $R$ such that

$$
\begin{aligned}
& R\left(\log (\rho), q \mid F, \overline{\log (\rho)}, \sigma_{\log (\rho)}, \gamma\right) \Delta \log (\rho) \Delta q \\
& \quad=\frac{\gamma+1}{\sqrt{2 \pi} \sigma} F q^{\gamma} \exp \left(-\frac{(\log (\rho)-\overline{\log (\rho)})^{2}}{2 \sigma_{\log (\rho)}^{2}}\right) \Delta q \Delta \log (\rho) .
\end{aligned}
$$

For this probability $R$, the corresponding value of $P(O \mid \theta)$ for our observed total number of companions $N_{\text {comp }}$ and total number of observations $N_{\mathrm{obs}}$ in that range of $(\Delta q, \Delta \log (\rho))$ is then given by the Poisson likelihood function:

$$
P\left(N_{\text {comp }}, N_{\text {obs }} \mid F, \overline{\log (\rho)}, \sigma_{\log (\rho)}, \gamma\right) \propto R^{N_{\text {comp }}} \times e^{-R \times N_{\text {obs }}} .
$$

We iterated our calculation over all mass ratios from 0 to 1 in steps of 0.01 and over all values of $\log (\rho)$ between 0.2 and 3.6 dex in steps of 0.1 dex, allowing each bin of $(\Delta q, \Delta \log (\rho))$ to serially modify the prior PDF and generate the posterior PDF.

As in our other work, we adopt several modifications to the formalism of A07. The most significant feature is to assume constant prior values for $F$ and $\sigma_{\log (\rho)}$, whereas the description in A07 suggests that he might have adopted priors proportional to $1 / F$ and $1 / \sigma_{\log (\rho)}$, respectively; we believe that the constant priors are more appropriate for an unbiased analysis with minimal initial assumptions. We chose to model the separation distribution in terms of observed projected separation rather than the underlying semimajor axis distribution. If the separations and eccentricities are uncorrelated, then the two distributions are related by a constant multiplier that depends on the eccentricity distribution (e.g., FM92), and we prefer to work with the observed quantity rather than an uncertain inferred quantity. We also omitted the flux-completeness correction used by A07 to compensate for the overluminosity of similar-brightness binaries. The discovery surveys for most of our sample members were spatially limited, not flux-limited, so binary systems were as likely to be detected as single stars. The detection limits of wavefront sensors in AO imaging are generally optically fluxlimited, but we chose our mass cutoff to alleviate this problem and have invoked the results of previous surveys to further account for any remaining incompleteness (Section 5; Table 6; 
Figure 3 ). Finally, our population parameter $F$ is more formally treated as a companion frequency (the number of companions per primary star) rather than a binary frequency (the number of primary stars with at least one companion). This distinction did not matter for the sample analyzed in A07 since it included no high-order multiple systems, but our sample includes many systems where one primary has more than one companion, and all of these companions contribute to the overall companion frequency per primary star.

Previous surveys have shown that the frequency and properties of multiple systems depend on the system mass (e.g., DM91 versus FM92), so we have conducted this analysis for the entire sample and for two subsets of primary masses: $0.7-2.5 M_{\odot}$ and $0.25-0.7 M_{\odot}$. The division between these subsets is located at the same mass as for our survey of Upper Scorpius, which will allow us to directly compare the results of our two surveys. However, our Taurus sample spans a wider total range of mass than that of our Upper Scorpius sample $\left(0.25-2.5 M_{\odot}\right.$ versus $\left.0.4-1.7 M_{\odot}\right)$, so any mass-dependent trends should be evaluated accordingly.

As we noted in the previous section, we also must consider an alternate separation distribution, which is a log-flat distribution with mass-dependent inner and outer cutoffs. However, we can only constrain one such cutoff across the separation range of our sample (the outer cutoff for lower-mass stars), so the results of Bayesian analysis for that model are trivially unconstrained. As we describe in the next subsection, we can use the results of our Bayesian analysis to estimate the completeness-corrected binary frequency for separations of 3-5000 AU, which allows us to directly estimate the companion frequency per decade of separation, and thus a relation between the total companion frequency and the interval spanned between the inner and outer cutoffs of a log-flat function.

\subsection{The Primordial Multiplicity of Solar-type Stars}

Our Bayesian analysis yields a PDF for all possible "models" that is defined across four dimensions $\left(F, \gamma, \overline{\log (\rho)}\right.$, and $\left.\sigma_{\log (\rho)}\right)$, so we cannot present the full results in a two-dimensional medium. However, any uncorrelated parameters can be presented separately without discarding information. This independence allows us to present the results as a series of lowerdimensional surfaces, where the PDF is integrated across the uncorrelated parameters in order to flatten its dimensionality. We have found that our constraints on $\overline{\log (\rho)}$ and $\sigma$ are strongly correlated, while our constraints on $\gamma$ are not correlated with any other parameters, so we present our results in terms of two planes $(F$ versus $\overline{\log (\rho)}$ and $F$ versus $\sigma)$ and one interval $(\gamma)$.

In Figure 6, we show our joint constraints on the companion frequency, mean separation, and standard deviation of the separation, inferred for the entire sample and then for the highmass and low-mass subsamples. The observed frequency of companions ( $\sim 70 \%$ in each mass range) placed a lower limit on the overall companion frequency. However, since there could be a significant number of companions inside or outside the survey detection limits, significantly higher frequencies are allowed. Our results even allow for companion frequencies of $>100 \%$, which would indicate a significant population of hierarchical multiple systems.

For the high-mass subsample, the nearly log-flat separation distribution yields a wide range of allowed values. There is no correlation between the most probable mean separation and the frequency, but the range of allowed mean separations is
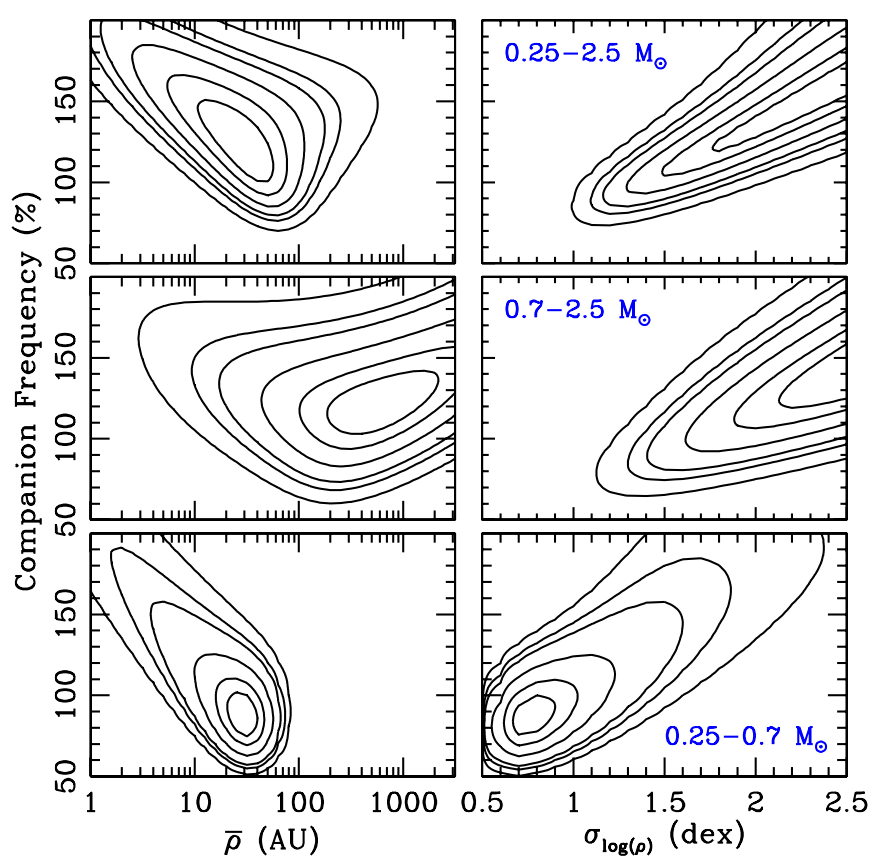

Figure 6. Joint constraints on the companion frequency vs. the mean (left) and standard deviation (right) of the separation distribution, as computed for the entire sample (top), only stars with $M>0.7 M_{\odot}$ (middle), and only stars with $M<0.7 M_{\odot}$ (bottom). Contours are drawn to enclose $25 \%, 50 \%, 75 \%$, $90 \%, 95 \%$, and $99 \%$ of the total probability. The apparently log-flat separation distribution for solar-type binaries is indicated by the wide range of possible mean separations and the strong tendency for large values of the standard deviation. For lower-mass binaries, the paucity of wide binary companions indicates that small values of the mean separation are preferred, though the unknown form of the distribution at separations $\lesssim 3$ AU yields a degeneracy between the companion frequency and mean separation. In both cases, the observed frequency of binary companions places a strong lower limit on the possible frequency ( $\gtrsim 60 \%-70 \%$ ).

(A color version of this figure is available in the online journal.)

correlated; we have observed that the companion frequency is $\sim 70 \%$ for separations of $\sim 3-5000 \mathrm{AU}$, so if the mean separation is not located at the logarithmic mean of this range ( $\sim 200 \mathrm{AU})$, then there must be additional companions beyond the inner or outer working angles of our survey. By similar reasoning, the standard deviation of the separation distribution is strongly correlated with the frequency. The nearly log-flat distribution for separations of 3-5000 AU indicates that additional companions (beyond those we observe) must be spread across a wide range of inner or outer separations, or else the distribution across our observed range would not appear flat.

For the low-mass subsample, the paucity of companions at separations of $\gtrsim 200$ AU clearly indicates a more restricted set of preferred models. If the companion frequency is significantly higher than the observed value of $\sim 70 \%$, then most of the additional companions must be found inside the inner working angle of our survey. As a result, a higher frequency is strongly correlated with a smaller mean and a larger standard deviation in the separation distribution. The relatively sharp outer limit in the binary population also weighs against significantly larger values of $\sigma_{\rho}$ (and thus higher frequencies) since an extended tail should not show such an abrupt decline.

In Figure 7, we show our confidence intervals for $\gamma$, again for the entire sample and for both subsamples. We have found that our constraints on $\gamma$ are not significantly correlated with the other parameters, a result of our survey's sensitivity to even very low mass ratios $(q<0.1)$ across most of its separation 


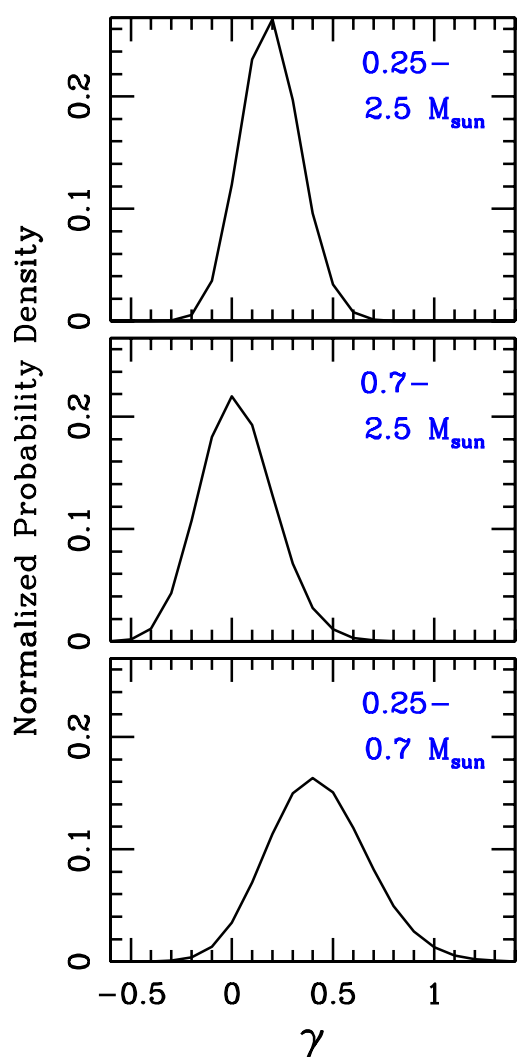

Figure 7. Confidence intervals for $\gamma$, the power-law exponent of the companion mass-ratio distribution. As for Figure 6, we show our results for the entire sample, only $M>0.7 M_{\odot}$, and only $M<0.7 M_{\odot}$. We find that all three cases yield the same value to within the uncertainties, and are typically consistent with a linear-flat case $(\gamma=0)$.

(A color version of this figure is available in the online journal.)

range. There is also little evidence for a mass dependence in the mass-ratio distribution. The estimated power-law slope for the entire sample, $\gamma=0.2 \pm 0.2$, is consistent at the $1 \sigma$ level with the values for the high-mass subsample $(\gamma=$ $0.0 \pm 0.2)$ and the low-mass subsample $(\gamma=0.4 \pm 0.2)$. However, studies of intermediate- to high-mass stars (2-10 $M_{\odot}$; Kouwenhoven et al. 2007) and VLM stars and brown dwarfs $\left(\lesssim 0.3 M_{\odot}\right.$; Burgasser et al. 2006; Allen 2007; A. Kraus \& L. A. Hillenbrand 2011, in preparation) demonstrate that a largescale trend does exist. The power-law slope for intermediateto high-mass stars is negative $(\gamma \sim-0.4$; Kouwenhoven et al. 2007), while the slope becomes increasingly negative near and below the substellar boundary $(\gamma \sim 2-4$; Kraus \& Hillenbrand 2010). We therefore suggest that either this entire mass range shows a gradual trend toward similar-mass companions at lower masses, or there are multiple processes that set the mass-ratio distribution in different primary mass ranges.

Interestingly, the DM91 mass function also appears to have a negative slope. They did not report a power-law fit, so we refit the histogram for their completeness-corrected $q$ distribution with a power law. We found that the entire distribution $(0<q<1.1)$ has a best-fit slope of $\gamma=-0.36 \pm 0.07$, albeit with a poor fit $\left(\chi_{v}=2.7\right.$ with 9 degrees of freedom $)$. If we omit the two lowestmass bins (which consist largely of completeness correction) and only fit the remaining range $(0.2<q<1.1)$, we find a much better fit ( $\chi_{v}=0.7$ with 7 degrees of freedom) and a much steeper negative slope of $\gamma=-1.2 \pm 0.2$. The former value disagrees with our entire sample at $\sim 3 \sigma$ and with our solar-type subsample at $\sim 2 \sigma$, while the latter value disagrees at $\sim 5 \sigma$ and $\sim 4 \sigma$, respectively. As we discuss below, this might reflect the application of too large a completeness correction for systems with low mass ratios, especially since updated surveys (e.g., Raghavan et al. 2010; Tokovinin 2011) also report a shallower mass-ratio distribution for field solar-type stars.

As we discussed in Section 1, the theoretical expectation is that binary systems with separations of $\gtrsim 100$ AU most likely form via free-fall fragmentation during early collapse, while systems with separations of $\lesssim 100$ AU most likely form via fragmentation of the protostellar disk after the primary had ceased free-fall collapse. These two processes occur at different times and should proceed in a very different fashion, so it seems plausible that they might produce binary companions with a different mass function. We tested for this difference by independently analyzing our results with the Bayesian formalism for the two separation ranges, then marginalizing the resulting PDFs to yield measurements and confidence intervals for $\gamma$; this yields PDFs like those shown for our mass subsamples in Figure 7. Contrary to our expectation, we find that there is no evidence for a different mass function at large separations than at small separations. The best-fit slope of the mass function is $\gamma=0.22 \pm 0.22$ at separations $\lesssim 100 \mathrm{AU}$ and $\gamma=0.08 \pm 0.20$ at separations $\gtrsim 100 \mathrm{AU}$, and hence the two mass functions are consistent to within $0.5 \sigma$.

Finally, we must conclude by explicitly noting the degeneracies in our parameter constraints that result from our survey's inner and outer working angles. The degeneracy due to the inner working angle will be broken by results from RV surveys, including ongoing programs by Prato et al. (2008), Nguyen et al. (2009), and R. White et al. (2011, in preparation). Breaking the degeneracy for extremely wide binary systems will be more difficult since binary systems must be distinguished from chance alignments of unbound stars (e.g., Kraus \& Hillenbrand 2008), but we are developing statistical tools for measuring and subtracting this contamination. In the meantime, we can avoid these degeneracies by forward modeling from our four-dimensional PDF back into the range of parameter space where our survey is mostly complete. This extrapolation is effectively a completeness correction that integrates the correction over all possible models, weighted by the probability of each model. To this end, we have integrated over the entire four-dimensional PDF of each mass subsample to extrapolate the binary frequency at separations of 3-5000 AU and spanning all mass ratios. We find that the total companion frequency in this range of parameter space is $64_{-9}^{+11} \%$ for $0.7-2.5 M_{\odot}$ stars and $79_{-11}^{+12} \%$ for $0.25-0.7 M_{\odot}$ stars.

\subsection{The Frequency of Single Stars}

It has been suggested that binary formation might be required for the vast majority of protostars because of the well-known problems with angular momentum dispersal (e.g., Bodenheimer 1995 and references therein). As we discussed above, our observational results alone are broadly consistent with this picture, with a total companion frequency of at least $\sim 65 \%-80 \%$. In this section, we will attempt to establish the frequency of single stars by estimating the frequency in the rest of parameter space, and ask if this is consistent with simple stochastic fragmentation models that neglect angular momentum considerations or feedback processes.

From our sample of 128 distinct, gravitationally bound systems (both binaries and singles), 11 systems are single to our knowledge but have no high-resolution observations, so 
we do not include them in the analysis of this section. Of the remaining 117 systems, 48 have no companions between 3 and $5000 \mathrm{AU}$, with detection limits reaching the substellar regime ( $q \lesssim 0.1$ or $M \lesssim 100 M_{\text {Jup }}$ ) in all cases and $\sim 10-20 M_{\text {Jup }}$ for the majority of targets. This places an upper limit of $41 \%$ for the single-star fraction of solar-type stars in Taurus. To arrive at a true single-star fraction over all separations, we must estimate the number of close and wide binaries that we have missed.

The DM91 distribution converted to apparent separation in $\mathrm{AU}$ (at a total mass of $1 M_{\odot}$ ) has a mean in $\log$ of 1.39 and a standard deviation of 1.53. According to this distribution, $27 \%$ of companions have separations smaller than 3 AU. Conservatively using the DM91 normalization of a $62 \%$ companion fraction for $q>0.1$ systems leaves us with 8 of the 48 apparently single stars with close companions. An extrapolation of our separation distribution with $\mu(\log (\rho))=$ 2.6, $\sigma(\log (\rho))=1.5$, and $F=1.05$ would give 14 apparently single stars with close companions. Two of these are the known spectroscopic binaries DQ Tau and V826 Tau.

For wide companions, we need to estimate the number of apparent binaries that are just chance alignments between nongravitationally bound Taurus members. Over the separation range of 30-120 arcsec, the characteristic surface density of unbound neighbors is $40 \mathrm{deg}^{-2}$ (Kraus \& Hillenbrand 2008), meaning that $6 \pm 2$ wide companions are actually just chance alignments. There are 10 apparent wide companions amongst our 48 apparently single stars, so we assume that $4 \pm 2$ of these are genuine physical companions. However, there could be additional undiscovered companions; several of these candidate ultrawide companions were discovered only within the past 1-2 years (Scelsi et al. 2008; Luhman et al. 2009).

Once close and wide systems are taken into account, we expect $\sim 30-38$ out of the 117 well-observed systems to be single, resulting in a single star fraction of $25 \%-32 \%$. Despite the higher multiplicity of Taurus stars compared to the field, even the lower end of this range shows that it is not uncommon for stars to form alone in a low-density environment such as Taurus.

Our Bayesian analysis determined a model for the probability density of companions as a function of separation and mass ratio. However, this does not directly determine the predicted fraction of single stars, or indeed the fraction of multiple systems of each order. In order to discuss these, we need to include prior constraints on some aspects of the semimajor axis and companion frequency probability distribution, because our data alone do not constrain the smallest and largest separations. We chose to use the projected separation distribution from Raghavan et al. (2010), with $\mu(\log (\rho))=1.6$ and $\sigma(\log (\rho))=1.5$. We have converted their period distribution to a projected separation distribution assuming a total mass of $1.5 M_{\odot}$ and assuming that projected separation is on average 0.8 times the semimajor axis (e.g., FM92). This distribution is very similar to that from DM91, and our discussion below is only weakly dependent on this prior assumption.

Using this separation distribution, our data give a companion fraction $F=1.15 \pm 0.15$ (Section 5.3; Figure 6). The simplest possible way to go from this probability density function to multiplicity is to assume that the likelihood of finding additional companions around a primary star at any separation is independent of previously found companions, and to neglect fragmentation of fragments. This would produce a multiplicity distribution which is a Poisson distribution with mean $F$. Such a simple assumption already gives a single star fraction of $32 \% \pm$
$5 \%$ and a sextuple or higher fraction of $0.5_{-0.2}^{+0.3} \%$, consistent with our single star fraction and the one sextuple system LkHa 332-G1 ABLkHa 332-G2AB/V955 Tau AB.

A slightly more sophisticated argument must involve the possibility of hierarchical fragmentation. We model this in a Monte Carlo method by treating our model probability density as a large-scale fragmentation probability. Each fragment can then re-fragment with the same probability density function, but only at a scale at least three times smaller than the previous fragmentation (to reflect the absence of stable orbits for nonhierarchical triples). The secondary has fragmentation suppressed with a probability of $25 \%$ in order to represent the possibility of a fragment falling below our mass limit for primaries in our sample $\left(M_{\text {frag }}<0.25 M_{\odot}\right.$; Section 2$)$, and fragmentation ceases if two fragments have a semimajor axis in the 1-4 arcsec range, to mimic our loss in sensitivity over this range (Section 2). This model results in the same single star fraction as the Poisson model $(32 \% \pm 5 \%)$, but a substantially higher sextuple or higher fraction of $4.5_{-1.7}^{+2.1} \%$. Restricting the separation distribution to our observed 3-5000 AU range gives a single star fraction of $37 \% \pm 5 \%$ and a sextuple (or higher) fraction of $2.3_{-0.7}^{+1.2} \%$.

This is consistent with the 48 apparently single stars and one quintuple or higher system in our sample of 117 stars. A more sophisticated treatment would need to take into account the mass dependence of fragmentation. With this extensive data set, we find that there is nothing unusual about single stars, as there would be if angular momentum evolution required the formation of binary companions as part of protostellar collapse. We also find the existence of high-order multiple systems in Taurus to be a natural product of fragmentation in a dynamically pristine environment.

\section{IMPLICATIONS FOR (MULTIPLE) STAR FORMATION}

Binary formation is expected to occur via two complementary pathways; wide ( $\gtrsim 100 \mathrm{AU}$ ) binary companions should form by fragmentation of the protostellar core during its initial free-fall collapse (e.g., Bodenheimer \& Burkert 2001), while close $(\lesssim 100$ AU) binary companion can form via gravitational instability and fragmentation in the protostellar accretion disk of the primary star (Toomre 1964; Boss 2001; Clarke 2009), most likely modified by subsequent migration through the disk. Both processes are ultimately based on the need for a collapsing protostar to dispel its angular momentum, but otherwise the detailed physics are quite distinct. As we describe below, only some of the predictions from theoretical models are verified by our observations, which suggests that these models remain incomplete.

A successful model for star formation should include these processes and successfully match the observed properties of the binary star population. The newest generation of theoretical models now match the slope and turnover of the IMF (e.g., Bate 2009a), but requiring simultaneous agreement with the (potentially mass-dependent) frequency, separation distribution, and mass-ratio distribution for binary systems is a far more demanding criterion, and one that has yet to be achieved. Any discrepancy with respect to observations will provide guidance in developing the next generation of models, marking the phenomena that might be lacking (i.e., radiative feedback or magnetic fields; Bate 2009b; Offner et al. 2009; Price \& Bate 2009) or overrepresented (dynamical interactions; Kraus \& Hillenbrand 2008, 2009a). 


\subsection{The Primordial Separation Distribution}

The overall separation distribution for young $(\lesssim 5 \mathrm{Myr})$ solar-type $\left(M \sim 0.7-2.5 M_{\odot}\right)$ stars in loose associations is significantly different from that observed in the field. Past surveys (e.g., DM91; Raghavan et al. 2010) have suggested that the field distribution is unimodal and log-normal, with a mean separation of $30 \mathrm{AU}$. In contrast, our results show that the separation distribution for solar-mass stars is approximately log-flat over at least 3.5 decades of separation (3-5000 AU), and our study of young star clustering (Kraus \& Hillenbrand 2008) suggests that the log-flat binary separation distribution might extend to at least 15,000 AU. The uniformity of the separation distribution for solar-mass binaries is quite surprising; as we described above and in the introduction, binary formation should occur through very different processes at very large and very small separations. The presence of a discontinuity in binary properties near the expected transition point ( $\sim 100 \mathrm{AU})$ would confirm this expectation. The lack of a discontinuity does not necessarily disprove the expectation, but it does argue that both modes yield similar results despite the very different evolutionary paths.

For wide $(\gg 100 \mathrm{AU})$ binary systems that are expected to fragment during or just after free-fall collapse, the initial semimajor axis of the binary system should depend primarily on the characteristic size of the core when fragmentation occurred and the location within the core where the critical overdensity was reached. Taurus hosts solar-type binary systems with separations of up to $\sim 10^{4} \mathrm{AU}$, which is similar to the characteristic size of prestellar cores in regions like the Pipe Nebula that could resemble the Taurus progenitor (Lada et al. 2008). This similarity suggests that fragmentation can occur very early, before the outer envelope has undergone significant free-fall collapse toward the central star. Observations of starless prestellar cores seem to indicate characteristic sizes of $\sim 10^{4} \mathrm{AU}$ (Menshchikov et al. 2010). Similar observations of more evolved Class 0 protostars seem to indicate that they have very large envelopes ( $\gtrsim 10^{3} \mathrm{AU}$; Looney et al. 2000) and condense from the inside out, with the outer envelope remaining unperturbed (aside from some rotational flattening) while the inner envelope undergoes obvious infall (e.g., Chiang et al. 2010). Observations of Class I protostars in Taurus indicate that the outer envelope radius remains large (Furlan et al. 2008), but as we discuss in the next subsection, most companions must fragment before the primary accretes most of the envelope mass. We therefore suggest that binary fragmentation must occur no later than the Class 0 stage, especially since many of the Class 0 systems observed by Looney et al. (2000) appear to have already fragmented into multiple widely separated components by their present age.

Timing constraints aside, it is unclear how Jeans-critical fragments could initially form in the outer regions of protostellar cores. Observations show that prestellar cores can be approximated by pressure-confined, thermally supported isothermal sphere (i.e., Bonnor 1956; Ward-Thompson et al. 1994) that have a density profile with approximately constant density for the inner $\sim 10^{3} \mathrm{AU}$ (where magnetic or turbulent support might dominate) and $\rho \propto r^{-2}$ at larger separations. In these cores, a Jeans-critical fragment would represent a far higher fractional overdensity at large radii as compared to small radii. The hydrodynamic models that are able to produce wide pairs (e.g., Delgado-Donate et al. 2004) usually start with a uniformdensity medium and allow the structure to emerge from free-fall and turbulent motions, but this is not consistent with the presence of quasi-stable pressure-confined cores even in pre-starforming environments like the Pipe Nebula (Lada et al. 2008). One solution might be for wide binary companions to fragment out of substructures that trace the larger structure of the starforming region. Stars seem to form along large-scale filaments in their progenitor giant molecular cloud (Goldsmith et al. 2008; Menshchikov et al. 2010), so if protostellar cores remain elongated as they collapse (as might be suggested by observations; Tobin et al. 2010), then wide companions could form more easily along the filament direction. An observational test of this hypothesis would be to observe wide binaries among Class 0 stars that are $\ll 1$ orbital period old, and thus determine whether their P.A. is aligned with the local filamentary structure of the star-forming region. Many regions have been surveyed in the MIR with Spitzer (e.g., Rebull et al. 2010) and will be observed in the submillimeter/millimeter with SCUBA-2 and ALMA, so this test could be feasible in the near future. Surveys of Class I binary systems (e.g., Connelley et al. 2008) might provide such a test, but most of the regions included in their sample have not been studied to characterize their larger-scale structure to the same extent as for Taurus (e.g., Goldsmith et al. 2008 for the gas or Kraus \& Hillenbrand 2008 for the stars).

It is also noteworthy that there are very few low-mass binary systems with very wide separations, but instead the separation distribution for lower-mass stars $\left(M_{\text {prim }}=0.25-0.7 M_{\odot}\right)$ appears to be truncated for separations of $\gtrsim 200 \mathrm{AU}$. This limit is similar to the limit seen in the field, where systems have been observed to follow a relation between the system mass $M$ and the maximum possible binary separation $a_{\max }$. The functional form of this trend is $a_{\max } \propto M^{2}$ for masses $<0.4 M_{\odot}$ (Burgasser et al. 2003), so the envelope corresponds to a constant binding energy at all masses. Previous studies have interpreted this binding energy cutoff as a signature of dynamical interactions, such that loosely bound systems are disrupted by interactions within the natal cluster. However, the interaction timescale in Taurus is much longer than its age, so external truncation by other stars does not seem to be a likely explanation. We therefore suggest that perhaps this correspondence with binding energy is coincidental across this regime, a point we made with respect to very wide binary systems in Kraus \& Hillenbrand (2009b), and that instead low-mass cores are simply unlikely to fragment during free-fall collapse. This hypothesis is consistent with new results for field multiple systems that are apparently too wide for their mass, as these systems often turn out to be hierarchical multiples with a higher total mass (e.g., Law et al. 2010), which seem to include many hierarchical multiples with higher total mass.

One alternate explanation which must be considered is that the outer separation limit is indeed dynamical in origin, but is set by the processes internal to the protostellar core and not by external stellar interactions. The most popular formulation of this concept is the "embryo ejection" model of star formation (Reipurth \& Clarke 2001), which postulates that protostellar cores produce many low-mass protostars or proto-brown dwarfs, but most are ejected from the protostellar envelope (and hence cut off from the reservoir of material to accrete) shortly after their fragmentation. This proposed mechanism originally seemed to be a natural complement to early gravoturbulent star formation simulations, which tended to fragment into dynamically active systems with many low-mass components. However, there have been ongoing debates regarding its observational predictions, particularly in the potential impact (or lack thereof) on the 
velocity and spatial distributions of star-forming regions (e.g., Luhman 2006; Kraus \& Hillenbrand 2008) and the frequency and properties of disks (e.g., White \& Basri 2003; Luhman 2004; Scholz et al. 2006). Recent updates to theoretical simulations may have solved these controversies; new model runs that incorporate magnetic fields and radiative feedback seem to produce systems with a few larger stars (Bate 2009b; Offner et al. 2009), rather than the many brown dwarfs seen in early models. We therefore suggest that even though some high- $N$ systems appear to form (such as V773 Tau and V955 Tau), the many-body outcome of star formation might not be a representative case for collapse and fragmentation of a typical protostellar core.

Given the prevalence of binary companions at small separations, disk fragmentation seems to remain a viable pathway for producing binary companions, so we instead suggest that the outer separation limit might indicate the maximum separation at which disk fragmentation can occur. For close ( $<100 \mathrm{AU})$ binary systems that are expected to form via disk fragmentation, the semimajor axis should depend on the radius at which fragmentation occurs and any subsequent migration of the binary companion. Disks are typically modeled using the formalism of $\alpha$-disk theory (Shakura \& Sunyaev 1973), which characterizes the viscosity as proportional to the local sound speed, vertical scale height, and a constant coefficient $\alpha$; the disk self-gravity is often modeled as a pseudoviscosity as well (e.g., Clarke 2009). The structure of early-stage protostellar disks is assumed to follow this model, but there are few observations that measure these disks' properties, so detailed predictions regarding the radius of initial fragmentation are not feasible yet. However, the most recent models predict that fragmentation should be most common in the outer portion of the disk ( 50-100 AU), where cooling is more efficient and orbital shear is less important (Matzner \& Levin 2005; Stamatellos et al. 2007).

We found many companions at separations down to $\sim 3 \mathrm{AU}$, so if the binary companions did not form in situ, then it seems likely that they formed at larger separations and migrated inward. Any companion that forms via disk instability should be large enough to open a gap immediately, so subsequent migration should proceed via the Type II mechanism (Lin \& Papaloizou 1985) and carry the companion inward; this tendency is borne out by simulations (Bate et al. 2002; Clarke 2009). The migration timescale depends on the primary and companion masses (being much longer for similar-mass companions), so it will depend on the accretion history of the system. For example, if the mass ratio is $1: 100$, then the migration timescale in a disk which is massive (10 times the minimum-mass solar nebula) and has a viscosity parameter of $\alpha=10^{-3}$ will be $10^{5} \mathrm{yr}$ at $1 \mathrm{AU}$ and $5 \times 10^{5}$ yr at $25 \mathrm{AU}$ (Ida \& Lin 2004). Increasing the mass ratio by a factor of 10 will lengthen the migration timescale by a factor of 10 , effectively freezing the companion at the location where significant accretion occurred. This suggests that the accretion history sets the final location of a companion, with the migration distance depending on the length of time before significant accretion occurs.

As we noted above, there are few low-mass binary systems $\left(M_{\text {prim }}=0.25-0.7 M_{\odot}\right)$ with separations of $\gtrsim 200 \mathrm{AU}$, but binary systems are very common at smaller separations. This discrepancy seems to indicate that disk fragmentation could be the preferred mechanism for low-mass binary formation, with little contribution from early fragmentation during free-fall collapse. Since migration typically moves companions inward, the mass-dependent maximum separation for binary systems
(Reid et al. 2001; Burgasser et al. 2003; Kraus \& Hillenbrand 2009a) could then be interpreted as denoting the separation regime at which disk fragmentation occurs: $\sim 100$ AU for earlyM stars, 20-30 AU for late-M stars, and 5-10 AU for brown dwarfs.

\subsection{The Mass-ratio Distribution}

Our observed mass-ratio distributions also do not agree with those of DM91, with a linear-flat primordial distribution in Taurus that features more similar-mass companions and fewer low-mass companions. There have been no processes suggested that would cause the field mass-ratio distribution to differ from the young (i.e., Class II) mass-ratio distribution, so given that DM91 relied on significant completeness corrections for the lowest-mass companions, we believe that the linear-flat distribution probably represents the true distribution in the field as well. As with the separation distribution, we did not expect the observed similarity of the mass-ratio distributions for wide companions ( $\gtrsim 100 \mathrm{AU})$ and very close companions ( $\lesssim 100 \mathrm{AU})$, so it is surprising that the different modes of binary formation yield similar results.

For wider binary pairs, fragmentation should cause the cloud to split into two separate core/envelope systems that then evolve independently, with some overlap of the envelopes for separations out to $\sim 10^{3} \mathrm{AU}$. In this case, the division of mass between the two components should be driven by their relative locations within the progenitor core and the specific angular momentum of the remaining envelope. In the case of pure freefall collapse, material should tend to accrete onto the nearer fragment, which would tend to yield a flat mass-ratio distribution if both positions are drawn at random. However, this should also yield a correlation such that wider binary systems have lower mass ratios, since fragments that initially form near the edge of the cloud will have access to less material to accrete. Conversely, if the material in the progenitor core has high specific angular momentum, then mass should preferentially accrete onto the star with the shallower potential well. This should drive the mass of the lower-mass secondary toward the mass of the primary, resulting in a mass ratio distribution that is peaked at unity.

Both of these results can be seen in hydrodynamic simulations (Bate 2000; Delgado-Donate et al. 2004; Bate 2009a). However, we find no preference for similar-mass companions in this mass range, and as we showed for wide binaries in Kraus \& Hillenbrand (2009a), even the widest systems have a flat mass ratio distribution. More detailed observations of very young protostars during the epoch of fragmentation (e.g., Duchêne et al. 2007) should cast more light on this process.

For close $(\lesssim 100 \mathrm{AU})$ binary pairs, the secondary fragments out of a circumprimary accretion disk and the two stars share a common envelope. Dynamical constraints on the total mass of a self-gravitating disk ensure that the initial mass of the secondary will be less than the primary mass, but the masses should be significantly altered by subsequent accretion out of the envelope, and will ultimately be set by the total mass remaining to be accreted, the specific angular momentum of the envelope material, and the orbital radius of the companion. As we described above, the initial orbital radius of the companion should be large ( $\sim 50-100 \mathrm{AU})$, but the radius will be modified (most likely inward) by migration and then frozen by additional accretion (e.g., Lin \& Papaloizou 1985; Bate et al. 2002; Ida \& Lin 2004). The relative accretion onto the primary or secondary will then be set by the orbital radius at which envelope material accretes onto the disk (Clarke 2009), which 
will be determined by the specific angular momentum and is observationally measured to also have a characteristic radius of 50-100 AU (Watson et al. 2007). Any material which falls outside the companion should accrete onto it, while material that falls inside the companion should accrete onto the primary. Some simulations suggest that the primary could see significant accretion even if the material has very high specific angular momentum (Ochi et al. 2005; Hanawa et al. 2010), though observations of systems with circumbinary disks tend to find that the secondary accretes more mass (e.g., Jensen et al. 2007).

Since observations indicate that envelope material accretes onto the disk at large radii, it seems likely that most of this material will accrete onto the secondary. The flat mass ratio distribution therefore indicates that at the time of companion fragmentation, the primary mass typically has approached its final mass and must constitute $\gtrsim 1 / 2$ of the entire core mass; if the envelope is accreted at a time-averaged constant rate until it is depleted, then fragmentation must occur with constant probability at any point after this limit, such that companions have a flat distribution of masses up to the primary mass. If the companion fragmented earlier in the envelope accretion stage, then competitive accretion would drive the mass ratio to unity, after which subsequent accretion would occur equally onto either component, yielding a significant population of "twins." Conversely, if the companions tended to fragment significantly later, then there would be insufficient material for a significant fraction of all companions to grow to similar masses as their primary stars. Clarke (2009) has suggested that the delay in companion fragmentation could result from an initially compact configuration for typical protostellar disks. Viscous evolution will transport angular momentum outward in a disk, so its outer radius might not spread outward into the regime where fragmentation can occur ( 50-100 AU) until after a significant amount of material has been accreted. However, this model relies on the envelope containing low specific angular momentum, such that most accretion occurs at small separations. The small number of detailed studies that measure envelope accretion (e.g., Watson et al. 2007) suggest that it actually occurs at the same characteristic radius as fragmentation.

\subsection{The Frequency of Protostellar Fragmentation}

The high companion frequency for Taurus has led to a persistent meme that in sparse environments, nearly all stars are born with binary companions. Newly formed stars must disperse a tremendous amount of angular momentum in condensing through $\sim 6$ orders of magnitude in radius, so binary formation would offer a convenient sink for much of this excess angular momentum. High multiplicity among solar-type stars would also match the predictions of many gravoturbulent star formation models, which tend to form small- $N$ clusters that subsequently evolve into a high-mass multiple system and many low-mass single stars and brown dwarfs. However, the assertion of nearuniversal primordial multiplicity has not been tested across the wide-separation range studied in our survey.

As we discuss in Section 5.4, it appears that $\sim 1 / 4-1 / 3$ of all star-forming cores that can form at least one $>0.25 M_{\odot}$ star will yield only that one star. Binary systems therefore cannot represent the only solution for overcoming the rotational support of angular momentum, though the single stars might represent the low-momentum tail of a natural distribution of total core angular momentum values. Our result shows that other processes like disk-locking (Königl 1991; Königl \& Pudritz 2000; Rebull et al. 2006) and winds (Shu et al. 2000; Matt \& Pudritz 2005) can be sufficient for dissipating a protostar's angular momentum. Our result also suggests that the dynamically active mode of gravoturbulent star formation has limited relevance to regions like Taurus; it is unlikely for solar-type stars to be rendered single in the decay of small- $N$ clusters (e.g., Goodwin \& Kroupa 2005), plus the low surface density and low velocity dispersion of Taurus members $\left(\Sigma \lesssim 5\right.$ stars $\mathrm{pc}^{-2}$ and $v \sim$ $200 \mathrm{~m} \mathrm{~s}^{-1}$; Kraus \& Hillenbrand 2008) indicate that often there are no small- $N$ clusters from which these stars could have been ejected.

Rather than universal multiplicity, the high companion frequency in Taurus is reflected by the highly multiple nature of some systems. In our subsample of 117 gravitationally bound systems with high angular resolution observations, there are 48 single stars, 50 binaries, 12 triples, 4 quadruples, and 1 sextuple when considering companions with separations of 3-5000 AU. The true multiplicities are higher than this: the J1-4872 system is a known quadruple, and the V807 Tau/GH Tau system is a known quintuple. However, one companion in each system was not included in our sample because we would not have been able to detect them with our survey, because of the AO "hole" for binary separations between 1 and 4 arcsec. As we showed in Section 5.4, our Monte Carlo companion distribution code successfully replicates the distribution of high-order multiples by assuming that once fragmentation has occurred, each component can fragment on smaller scales at the same rate as a similar-mass core that had not fragmented. For comparison, our code predicts $5.9 \pm 2.4$ quintuple or higher order systems, once all completeness corrections are taken into account. This number would be even higher if fragmentation of systems with primary masses $<0.25 M_{\odot}$ would be included. This is clearly higher than the number of high-order multiples expected in a field population, and suggests that in typical clustered star formation environments, high-order multiples do not survive because wide pairs tend to be broken apart (e.g., Reipurth et al. 2007; Kraus \& Hillenbrand 2009a).

\section{SUMMARY}

We have conducted a high-resolution imaging study of the multiple star population in the Taurus-Auriga star-forming region. Our results have significant implications for the primordial outcome of multiple star formation. To summarize:

1. We have identified 16 additional binary companions to primary stars with masses of $0.25-2.5 M_{\odot}$, raising the total number of companions at separations $0^{\prime \prime} 015-30^{\prime \prime}$ to 90 . Combined with our previous survey of wide binary systems, we have now compiled a comprehensive census spanning separations of 3-5000 AU.

2. We have found that $\sim 2 / 3-3 / 4$ of all Taurus targets are multiple systems of two or more stars, while the other $\sim 1 / 4$ $1 / 3$ appear to have formed as single stars. The distribution of high-order multiples is consistent with fragmentation occurring independently on all scales; once a collapsing protostellar core has fragmented into two components, either component can further fragment with the same probability as a single star of the same mass.

3. For solar-type stars $\left(0.7-2.5 M_{\odot}\right)$, the separation distribution is very nearly log-flat over separations of 3-5000 AU. In contrast, lower-mass stars $\left(0.25-0.7 M_{\odot}\right)$ show a paucity of binary companions with separations of $\gtrsim 200$ AU. Across this full mass range, the companion mass function is well described as a linear-flat function; all companion mass 
ratios are equally probable, apparently including substellar companions.

4. Binary formation on large scales ( $\gtrsim 100-200 \mathrm{AU})$ probably occurs via fragmentation during initial free-fall collapse, so the existence of wide companions (extending to $\sim 10^{4} \mathrm{AU}$ ) indicates that fragmentation can occur very early. We suggest that these companions might find their origin in the traces of larger-scale structure within the cloud, as a spherically symmetric isothermal sphere should not easily fragment on these scales, and if it did, it would preferentially form lower-mass companions. The paucity of wider companions to low-mass primaries might indicate that low-mass protostellar cores do not fragment during free-fall collapse.

5. Binary formation on smaller scales $(\lesssim 100-200$ AU) probably occurs via fragmentation of the protostellar accretion disk that forms after free-fall collapse has ended. Fragmentation should occur in the outer disk ( $\gtrsim 50 \mathrm{AU})$, so the log-flat separation distribution must indicate how far these companions migrate inward before they grow too massive to migrate. The flat mass ratio distribution seems to indicate that fragmentation occurs during the last half of envelope accretion. If fragmentation occurred while most mass was still in the envelope, then competitive accretion would drive system mass ratios preferentially to unity. Conversely, if most fragmentation occurred late, then the envelope would lack sufficient mass to grow $\sim 1 / 2$ of all companions to within $\gtrsim 1 / 2$ of the primary mass.

We thank Peter Tuthill and Jamie Lloyd for campaigning to have aperture masks installed in PHARO and NIRC2. We also thank Russel White for sharing the results of his multiplicity survey in Taurus prior to publication. Finally, we thank the referee for a detailed critique of this paper. A.L.K. has been supported by a SIM Science Study and by NASA through Hubble Fellowship grant 51257.01 awarded by STSCI, which is operated by AURA, Inc., for NASA, under contract NAS 5-26555. M.J.I. is supported by an Australian Postdoctoral fellowship from the Australian Research Council. This work makes use of data products from 2MASS, which is a joint project of the University of Massachusetts and IPAC/Caltech, funded by NASA and the NSF. Our research has also made use of the USNOFS Image and Catalogue Archive operated by the United States Naval Observatory, Flagstaff Station (http://www.nofs.navy.mil/data/fchpix/).

We recognize and acknowledge the very significant cultural role and reverence that the summit of Mauna Kea has always had within the indigenous Hawaiian community. We are most fortunate to have the opportunity to conduct observations from this mountain.

\section{REFERENCES}

Allen, P. 2007, ApJ, 668, 492 (A07)

Baraffe, I., Chabrier, G., Allard, F., \& Hauschildt, P. H. 1998, A\&A, 337, 403 Baraffe, I., Chabrier, G., Barman, T., Allard, F., \& Hauschildt, P. 2003, A\&A, 402,701

Bate, M. 2000, MNRAS, 314, 33

Bate, M. 2009a, MNRAS, 392, 590

Bate, M. 2009b, MNRAS, 392, 1363

Bate, M., Bonnell, I., \& Bromm, V. 2002, MNRAS, 336, 705

Boden, A., et al. 2007, ApJ, 670, 1214

Bodenheimer, P. 1995, ARA\&A, 33, 199

Bodenheimer, P., \& Burkert, A. 2001, in IAU Symp. 200, The Formation of Binary Stars, ed. H. Zinnecker \& R. D. Mathieu (San Francisco, CA: ASP), 13
Bonnell, I. 1994, MNRAS, 269, 837

Bonnell, I. 2001, in IAU Symp. 200, The Formation of Binary Stars, ed. H. Zinnecker \& R. D. Mathieu (San Francisco, CA: ASP), 23

Bonnor, W. 1956, MNRAS, 116, 351

Boss, A. 2001, ApJ, 551, 167

Bouvier, J., Rigaut, F., \& Nadeau, D. 1997, A\&A, 323, 139

Bouy, H., et al. 2003, AJ, 126, 1526

Briceño, C., Calvet, N., Kenyon, S., \& Hartmann, L. 1999, AJ, 118, 1354

Briceño, C., Hartmann, L., Stauffer, J., \& Martín, E. 1998, AJ, 115, 2074

Briceño, C., et al. 2002, ApJ, 580, 317

Burgasser, A., Kirkpatrick, J. D., Reid, I. N., Brown, M., Miskey, C., \& Gizis, J. 2003, ApJ, 586, 512

Burgasser, A., et al. 2006, ApJS, 166, 585

Carpenter, J. 2001, AJ, 121, 2851

Chabrier, G., Baraffe, I., Allard, F., \& Hauschildt, P. 2000, ApJ, 542, 464

Chen, W., Simon, M., Longmore, A., Howell, R., \& Benson, J. 1990, ApJ, 357, 224

Chiang, H.-S., Looney, L., Tobin, J., \& Hartmann, L. 2010, ApJ, 709, 470

Clarke, C. 2009, MNRAS, 396, 1066

Close, L., et al. 2003, ApJ, 587, 407

Connelley, M., Reipurth, B., \& Tokunaga, A. 2008, AJ, 135, 2496

Correia, S., Zinnecker, H., Ratzka, Th., \& Sterzik, M. 2006, A\&A, 459, 909

D’Antona, F., \& Mazzitelli, I. 1997, Mem. Soc. Astron. Ital., 68, 807

Delgado-Donate, E., Clarke, C., Bate, M., \& Hodgkin, S. 2004, MNRAS, 351, 617

Duchêne, G., Bontemps, S., Bouvier, J., Andre, P., Djupvik, A., \& Ghez, A. 2007, A\&A, 476, 229

Duchêne, G., McCabe, C., Ghez, A., \& Macintosh, B. 2004, ApJ, 606, 969

Duchêne, G., Monin, J.-L., Bouvier, J., \& Menard, F. 1999, A\&A, 351, 954

Duchêne, G., et al. 2010, ApJ, 712, 112

Duquennoy, A., \& Mayor, M. 1991, A\&A, 248, 485 (DM91)

Durisen, R., Gingold, R., Tohline, J., \& Boss, A. 1986, ApJ, 305, 281

Enoch, M., Evans, N., Sargent, A., \& Glenn, J. 2009, ApJ, 692, 973

Fischer, D., \& Marcy, G. 1992, ApJ, 396, 178 (FM92)

Fortney, J., Marley, M., Saumon, D., \& Lodders, K. 2008, ApJ, 683, 1104

Furlan, E., et al. 2008, ApJS, 176, 184

Ghez, A., Neugebauer, G., \& Matthews, K. 1993, AJ, 106, 2005

Ghez, A., White, R., \& Simon, M. 1997, ApJ, 490, 353

Ghez, A., et al. 2008, ApJ, 689, 1044

Goldsmith, P., Heyer, M., Narayanan, G., Snell, R., Li, D., \& Brunt, C. 2008, ApJ, 680, 428

Goodwin, S., \& Kroupa, P. 2005, A\&A, 439, 565

Grether, D., \& Lineweaver, C. 2006, ApJ, 640, 1051

Haisch, K., Greene, T., Barsony, M., \& Stahler, S. 2004, AJ, 127, 1747

Hanawa, T., Ochi, Y., \& Ando, K. 2010, ApJ, 708, 485

Hartigan, P., \& Kenyon, S. 2003, ApJ, 583, 334

Hartigan, P., Strom, K., \& Strom, S. 1994, ApJ, 427, 961

Hayward, T., Brandl, B., Pirger, B., Blacken, C., Gull, G., Schoenwald, J., \& Houck, J. 2001, PASP, 113, 105

Hillenbrand, L., \& White, R. 2004, ApJ, 604, 741

Ida, S., \& Lin, D. 2004, ApJ, 604, 388

Ireland, M., \& Kraus, A. 2008, ApJ, 688, L59

Ireland, M., Kraus, A., Martinache, F., Lloyd, J., \& Tuthill, P. 2008, ApJ, 678, 463

Itoh, Y., et al. 2005, ApJ, 620, 984

Jensen, E., et al. 2007, AJ, 134, 241

Kenyon, S., Brown, D., Tout, C., \& Berlind, P. 1998, AJ, 115, 2491

Kenyon, S., \& Hartmann, L. 1995, ApJS, 101, 117

Köhler, R., Kunkel, M., Leinert, C., \& Zinnecker, H. 2000, A\&A, 356, 541

Köhler, R., Petr-Gotzens, M., McCaughrean, M., Bouvier, J., Duchêne, G., Quirrenbach, A., \& Zinnecker, H. 2006, A\&A, 458, 461

Königl, A. 1991, ApJ, 370, 39

Königl, A., \& Pudritz, R. 2000, in Protostars and Planets IV, ed. V. Mannings,

A. P. Boss, \& S. S. Russell (Tucson, AZ: Univ. Arizona Press), 750

Konopacky, Q., Ghez, A., Rice, E., \& Duchêne, G. 2007, ApJ, 663, 394

Kouwenhoven, M., Brown, A., \& Kaper, L. 2007, A\&A, 464, 581

Kraus, A., \& Hillenbrand, L. 2007a, ApJ, 662, 413

Kraus, A., \& Hillenbrand, L. 2007b, AJ, 134, 2340

Kraus, A., \& Hillenbrand, L. 2008, ApJ, 686, 111

Kraus, A., \& Hillenbrand, L. 2009a, ApJ, 703, 1511

Kraus, A., \& Hillenbrand, L. 2009b, ApJ, 704, 531

Kraus, A., Ireland, M., Martinache, F., \& Lloyd, J. 2008, ApJ, 679, 762 (K08)

Kraus, A., et al. 2011, ApJ, submitted

Lada, C., Muench, A., Rathborne, J., Alves, J., \& Lombardi, M. 2008, ApJ, 672, 410

Law, N., Dhital, S., Kraus, A., Stassun, K., \& West, A. 2010, ApJ, 720, 1727

Leinert, Ch., et al. 1993, A\&A, 278, 129 
Lin, D., \& Papaloizou, J. 1985, in Protostars and Planets II, ed. D. C. Black \& M. S. Matthews (Tucson, AZ: Univ. Arizona Press), 981

Lloyd, J., Martinache, F., Ireland, M., Monnier, J., Pravdo, S., Shaklan, S., \& Tuthill, P. 2006, ApJ, 650, 131

Loinard, L., et al. 2007, ApJ, 671, 546

Looney, L., Mundy, L., \& Welch, W. 2000, ApJ, 529, 477

Luhman, K. 2000, ApJ, 544, 1044

Luhman, K. 2004, ApJ, 617, 1216

Luhman, K. 2006, ApJ, 645, 676

Luhman, K., Briceño, C., Stauffer, J., Hartmann, L., Barrado y Navascues, D., \& Caldwell, N. 2003a, ApJ, 590, 348

Luhman, K., Mamajek, E., Allen, P., \& Cruz, K. 2009, ApJ, 703, 399

Luhman, K., \& Rieke, G. 1998, ApJ, 497, 354

Luhman, K., Stauffer, J., Muench, Al, Rieke, G., Lada, E., Bouvier, J., \& Lada, C. 2003b, ApJ, 593, 1093

Luhman, K., Whitney, B., Meade, M., Babler, B., Indebetouw, R., Bracker, S., \& Churchwell, E. 2006, ApJ, 647, 1180

Marcy, G., \& Butler, R. 2000, PASP, 112, 137

Marley, M., Fortney, J., Hubickyj, O., Bodenheimer, P., \& Lissauer, J. 2007, ApJ, 655,541

Martín, E. 2000, AJ, 120, 2114

Martinache, F., Lloyd, J., Ireland, M., Yamada, R., \& Tuthill, P. 2007, ApJ, 661, 496

Martinache, F., Rojas-Ayala, B., Ireland, M., Lloyd, J., \& Tuthill, P. 2009, ApJ, 695,1183

Massarotti, A., Latham, D., Torres, G., Brown, R., \& Oppenheimer, B. 2005, AJ, 129,2294

Mathieu, R., Stassun, K., Basri, G., Jensen, E., Johns-Krull, C., Valenti, J., \& Hartmann, L. 1997, AJ, 113, 1841

Mathieu, R., Walter, F., \& Myers, P. 1989, AJ, 98, 987

Matt, S., \& Pudritz, R. 2005, ApJ, 632, 135

Matzner, C., \& Levin, Y. 2005, ApJ, 628, 817

Menshchikov, A., et al. 2010, A\&A, 518, L103

Metchev, S., \& Hillenbrand, L. 2009, ApJS, 181, 62

Nakajima, T., et al. 1989, AJ, 97, 1510

Nguyen, D., Jayawardhana, R., van Kerkwijk, M., Brandeker, A., Scholz, A., \& Damjanov, I. 2009, ApJ, 695, 1648

Ochi, Y., Sugimoto, K., \& Hanawa, T. 2005, ApJ, 623, 922

Offner, S., Klein, R., McKee, C., \& Krumholz, M. 2009, ApJ, 703, 131

Patience, J., Ghez, A., Reid, I. N., \& Matthews, K. 2002, AJ, 123, 1570

Prato, L., Huerta, M., Johns-Krull, C., Mahmud, N., Jaffe, D., \& Hartigan, P. 2008, ApJ, 687, 103

Prato, L., Simon, M., Mazeh, T., Zucker, S., \& McLean, I. 2002, ApJ, 579, 99

Price, D., \& Bate, M. 2009, MNRAS, 398, 33

Raghavan, D., et al. 2010, ApJS, 190, 1

Ratzka, Th., et al. 2009, A\&A, 502, 623

Readhead, A., Nakajima, T., Larson, T., Neugebauer, G., Oke, J., \& Sargent, W. 1988, AJ, 95, 1278

Rebull, L., Stauffer, J., Megeath, S., Hora, J., \& Hartmann, L. 2006, ApJ, 646, 297

Rebull, L., et al. 2010, ApJS, 186, 259
Reid, I. N., \& Gizis, J. 1997, AJ, 113, 2246

Reid, I. N., Gizis, J., Kirkpatrick, J. D., \& Koerner, D. 2001, AJ, 121, 489

Reipurth, B., \& Clarke, C. 2001, AJ, 122, 432

Reipurth, B., Guimarães, M., Connelley, M., \& Bally, J. 2007, AJ, 134, 2272

Richichi, A., Ragland, S., \& Fabbroni, L. 1998, A\&A, 330, 578

Sartoretti, P., Brown, R., Latham, D., \& Torres, G. 1998, A\&A, 334, 592

Scelsi, L., Sacco, G., Affer, L., Argiroffi, C., Pillitteri, I., Maggio, A., \& Micela, G. 2008, A\&A, 490, 601

Schaefer, G., Dutrey, A., Guilloteau, S., Simon, M., \& White, R. 2009, ApJ, 701, 698

Scholz, A., Jayawardhana, R., \& Wood, K. 2006, ApJ, 645, 1498

Schmidt-Kaler, Th. 1982, Physical Parameters of the Stars, Landolt-Bornstein Numerical Data and Functional Relationships in Science and Technology, New Series, Group VI, Volume 2b (Berlin: Springer)

Shakura, N., \& Sunyaev, R. 1973, A\&A, 24, 337

Shu, F., Najita, J., Shang, H., \& Li, Z.-Y. 2000, in Protostars and Planets IV, ed. V. Mannings, A. P. Boss, \& S. S. Russell (Tucson, AZ: Univ. Arizona Press), 789

Simon, M., Beck, T., Greene, T., Howell, R., Lumsden, S., \& Prato, L. 1999, AJ, 117,1594

Simon, M., Holfeltz, S., \& Taff, L. 1996, ApJ, 469, 890

Simon, M., et al. 1995, ApJ, 443, 625

Slesnick, C., Carpenter, J., Hillenbrand, L., \& Mamajek, E. 2006, AJ, 132, 2665

Stamatellos, D., Hubber, D., \& Whitworth, A. 2007, MNRAS, 382, 30

Stapelfeldt, K., Menard, F., Watson, A., Krist, J., Dougados, C., Padgett, D., \& Brandner, W. 2003, ApJ, 589, 410

Steffen, A., et al. 2001, AJ, 122, 997

Strom, K., \& Strom, S. 1994, ApJ, 424, 237

Tanner, A., et al. 2007, PASP, 119, 747

Tobin, J., Hartmann, L., Looney, L., \& Chiang, H.-F. 2010, ApJ, 712, 1010

Tohline, J. 2002, ARA\&A, 40, 349

Tokovinin, A. 2011, AJ, 141, 52

Toomre, A. 1964, ApJ, 139, 1217

Torres, R., Loinard, L., Mioduszewski, A., \& Rodrìguez, L. 2007, ApJ, 671, 1813

Torres, R., Loinard, L., Mioduszewski, A., \& Rodrìguez, L. 2009, ApJ, 698, 242

Tuthill, P., Monnier, J., Danchi, W., Wishnow, E., \& Haniff, C. 2000, PASP, 112,555

Tuthill, P., et al. 2006, Proc. SPIE, 6272, 62723A

Ward-Thompson, D., Scott, F., Hills, R., \& Andre, P. 1994, MNRAS, 268, 276

Watson, D., et al. 2007, Nature, 448, 1026

White, R., \& Basri, G. 2003, ApJ, 582, 1109

White, R., \& Ghez, A. 2001, ApJ, 556, 265

White, R., Ghez, A., Reid, I. N., \& Schultz, G. 1999, ApJ, 520, 811

White, R., \& Hillenbrand, L. 2004, ApJ, 616, 998

White, R., \& Hillenbrand, L. 2005, ApJ, 621, 65

Woitas, J., Köhler, R., \& Leinert, C. 2001, A\&A, 369, 249

Woitas, J., \& Leinert, C. 1998, A\&A, 338, 122

Zacharias, N., Monet, D. G., Levine, S. E., Urban, S. E., Gaume, R., \& Wycoff, G. L. 2004, BAAS, 205, 48.15 